\title{
Towards a 3-D tomographic retrieval for the air-borne limb-imager GLORIA
}

\author{
J. Ungermann ${ }^{1,2}$, M. Kaufmann ${ }^{1}$, L. Hoffmann ${ }^{1}$, P. Preusse ${ }^{1}$, H. Oelhaf ${ }^{3}$, F. Friedl-Vallon ${ }^{3}$, and M. Riese ${ }^{1}$ \\ ${ }^{1}$ Institut für Chemie und Dynamik der Geosphäre (ICG-1), Forschungszentrum Jülich, Jülich, Germany \\ ${ }^{2}$ German Research School for Simulation Sciences GmbH, Jülich, Germany \\ ${ }^{3}$ Institut für Meteorologie und Klimaforschung, Karlsruher Institut für Technologie, Karlsruhe, Germany
}

Received: 8 June 2010 - Published in Atmos. Meas. Tech. Discuss.: 15 July 2010

Revised: 3 November 2010 - Accepted: 17 November 2010 - Published: 29 November 2010

\begin{abstract}
GLORIA (Gimballed Limb Observer for Radiance Imaging of the Atmosphere) is a new remote sensing instrument essentially combining a Fourier transform infrared spectrometer with a two-dimensional (2-D) detector array in combination with a highly flexible gimbal mount. It will be housed in the belly pod of the German research aircraft HALO (High Altitude and Long Range Research Aircraft). It is unique in its combination of high spatial and state-ofthe art spectral resolution. Furthermore, the horizontal view angle with respect to the aircraft flight direction can be varied from $45^{\circ}$ to $135^{\circ}$. This allows for tomographic measurements of mesoscale events for a wide variety of atmospheric constituents.

In this paper, a tomographic retrieval scheme is presented, which is able to fully exploit the manifold radiance observations of the GLORIA limb sounder. The algorithm is optimized for massive 3-D retrievals of several hundred thousands of measurements and atmospheric constituents on common hardware. The new scheme is used to explore the capabilities of GLORIA to sound the atmosphere in full 3-D with respect to the choice of the flightpath and to different measurement modes of the instrument using ozone as a test species. It is demonstrated that the achievable resolution should approach $200 \mathrm{~m}$ vertically and $20 \mathrm{~km}-30 \mathrm{~km}$ horizontally. Finally, a comparison of the 3-D inversion with conventional 1-D inversions using the assumption of a horizontally homogeneous atmosphere is performed.
\end{abstract}

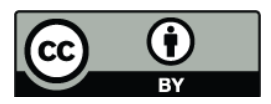

Correspondence to: J. Ungermann (j.ungermann@fz-juelich.de)

\section{Introduction}

Trace gas exchange between troposphere and stratosphere greatly contributes to upper troposphere and lower stratosphere (UTLS) composition changes and variability. Changes and variability of composition in the UTLS are, in turn, major drivers of surface climate change (e.g. Forster and Shine, 1997). Stevenson et al. (2006) highlight large model differences in the representation of stratosphere/troposphere exchange that lead to considerable uncertainties in the budget of ozone in the upper troposphere. Solomon et al. (2010) recently noted that global models are rather limited in their representation of key processes that determine the distribution and variability of water vapor in the lower stratosphere (such as stratosphere-troposphere-exchange). Consequently, these models are unable to reproduce long-term variations of water vapor in the lower stratosphere that turn out to be an important driver of decadal global surface climate change. These findings point to the need of a better quantitative understanding of processes in the UTLS (operating at different scales) that influence the spatial distribution of important greenhouse substances such as water vapor and ozone.

In the past, most progress in our understanding of UTLS processes has been made by detailed in-situ airborne measurements (e.g. Hoor et al., 2004; Schiller et al., 2009) or limb-sounding satellite instruments (e.g. Hegglin et al., 2009). However, airborne observations and current limbobservations by satellites are rather limited in terms of coverage and/or spatial resolution. This limitation may be overcome in the future by new limb imaging concepts (e.g. Riese et al., 2005; ESA, 2008; Ungermann et al., 2010), which will greatly improve vertical sampling as well as the horizontal sampling and coverage, thereby providing an unprecedented view on meso-scale structures (e.g. filaments, tropopause folds, mixing etc.) that play a crucial role for UTLS composition and variability (Konopka et al., 2009). 
In particular, limb-emission measurement techniques provide high spatial resolution and good coverage at the same time. Trace gas fields obtained from global infrared limbemission sounding (e.g. LIMS; Gille and Russel III, 1984; SAMS; Drummond et al., 1980; CRISTA; Riese et al., 1997; Offermann et al., 1999; or MIPAS; Fischer et al., 2007) greatly contributed to our understanding of the 3-D composition, structure and large-scale dynamics of the middle atmosphere (e.g. Riese et al., 2002). However, the spatial resolution obtained even by these sensors is relatively coarse compared to the scales of structures in the tropopause region and must by adequately improved in the future (e.g. ESA, 2008). Current airborne limb-emission sensors such as MIPAS-STR (e.g. Höpfner et al., 2001; Keim et al., 2008) and CRISTANF (e.g. Spang et al., 2008; Hoffmann et al., 2009) on-board the high-flying Russian aircraft Geophysica provide adequate spatial sampling in two dimensions (vertical and along the flight track). However, they are limited by a relative broad weighting function (about $200 \mathrm{~km}$ ) of the observations in the viewing direction (perpendicular to the flight track).

Where such conventional remote sensing instruments cannot resolve the atmosphere well along line-of-sight, the new Gimballed Limb Observer for Radiance Imaging of the Atmosphere (GLORIA) instrument has the capability to derive, for the first time, fine atmospheric structures in three dimensions. GLORIA is a development of Forschungszentrum Jülich $\mathrm{GmbH}$ and the Karlsruhe Institute for Technology. Deriving highly resolved 3-D temperature and trace gas concentration fields from GLORIA observations (e.g. for transport studies) poses a tomographic problem that needs special attention to handle the computational complexity. Further, GLORIA serves as an air-borne precursor for a proposed satellite-borne instrument (ESA, 2008).

2-D tomographic retrievals for satellite-borne, limbsounding measurements were explored by a variety of authors for different purposes and individual instruments (e.g. Solomon et al., 1984; Gurevich, 1995). Practical implementations for the large-scale 2-D tomographic retrieval of atmospheric constituents were first produced by Carlotti et al. (2001) for MIPAS and Livesey and Read (2000) for MLS (Microwave Limb Sounder). However, no current satellite infrared limb-sounder can deliver a highly resolved 3-D picture of atmospheric structures. Further, due to the different observations geometries between forward- or backwardlooking satellite instruments and side-ways looking airborne instruments, the posed 3-D tomographic problem is quite different from state-of-the art 2-D tomographic retrievals (Carlotti et al., 2006; Livesey et al., 2006). It is therefore not a simple extension from the simpler 2-D tomographic retrievals and its characteristics may be vastly different.

In Sect. 2, we introduce the GLORIA instrument in more detail. We present our methodology for tomographic retrievals in Sect. 3, thereby first describing our forward model in Sect. 3.1 and our regularization scheme in Sect. 3.2. Some important implementation details for handling the
Table 1. This table shows the principal technical characteristics of the GLORIA instrument. The panning angle might be slightly reduced depending on the aircraft onto which GLORIA will be mounted. Both spectral resolution and NESR are given for apodized spectra.

\begin{tabular}{lll}
\hline \multicolumn{2}{c}{ GLORIA instrument specification $(15 \mathrm{~km}$ observer altitude $)$} \\
\hline Mode & Dynamics & Chemistry \\
\hline lower limit of FOV & $-3.27^{\circ}(4 \mathrm{~km})$ & $-3.27^{\circ}(4 \mathrm{~km})$ \\
upper limit of FOV & $+0.8^{\circ}$ & $+0.8^{\circ}$ \\
horizontal FOV & $4.07^{\circ}$ & $4.07^{\circ}$ \\
vertical sampling & $140 \mathrm{~m}$ & $560 \mathrm{~m}$ \\
horizontal sampling & $2.2 \mathrm{~km}$ & $17.9 \mathrm{~km}$ \\
panning & $45^{\circ}-135^{\circ}$ & $90^{\circ}$ \\
spectral coverage & $770-1400 \mathrm{~cm}^{-1}$ & $770-1400 \mathrm{~cm}^{-1}$ \\
spectral resolution & $1.25 \mathrm{~cm}^{-1}$ & $0.1 \mathrm{~cm}^{-1}$ \\
NESR & $3.5 \mathrm{nW} /\left(\mathrm{cm}^{2} \mathrm{srcm}^{-1}\right)$ & $2 \mathrm{nW} /\left(\mathrm{cm}^{2} \mathrm{srcm}^{-1}\right)$ \\
acquisition time & $\approx 3 \mathrm{~s}$ & $\approx 36 \mathrm{~s}$ \\
\hline
\end{tabular}

sheer amount of unknowns and measurements are presented in Sect. 3.3 with respect to the inversion and in Sect. 3.4 with respect to diagnosing the results. We apply the methodology on a simplified retrieval setup and analyze the principal capabilities of a tomographic retrieval using simulated measurements of GLORIA in Sects. 4.1 to 4.4. We finally compare the results with conventional retrievals using the assumption of horizontal homogeneity in Sect. 4.5.

\section{The GLORIA infrared limb imager}

GLORIA (Fig. 1) is an infrared remote limb sounder that provides unprecedented 3-D sampling of the atmosphere for a limb sounder. Infrared limb sounding exploits the radiation thermally emitted in the atmosphere along the line-ofsight (LOS) of the instrument, which is directed towards the limb of the Earth's atmosphere. The point of the LOS closest to the surface is called the tangent point. Under opticallythin conditions, the tangent point of the LOS generally determines the point in the atmosphere that contributes most to the measured radiation.

The GLORIA instrument (Friedl-Vallon et al., 2006) essentially combines a Fourier transform infrared spectrometer with a two-dimensional (2-D) detector array measuring in the spectral regions from $770 \mathrm{~cm}^{-1}$ to $1400 \mathrm{~cm}^{-1}$ (see Table 1 for instrument characteristics). The 2-D detector array will consist of about $128 \times 128$ pixels, providing more than 16000 simultaneous limb-views within an elevation angle of $-3.27^{\circ}$ (at an observer altitude of $15 \mathrm{~km}$ this corresponds to a tangent altitude of roughly $4 \mathrm{~km}$ the tangent point being $\approx 375 \mathrm{~km}$ away from the observer) to $0.8^{\circ}$ and with a horizontal field-of-view of $4.07^{\circ}$. To increase the signal-to-noise 


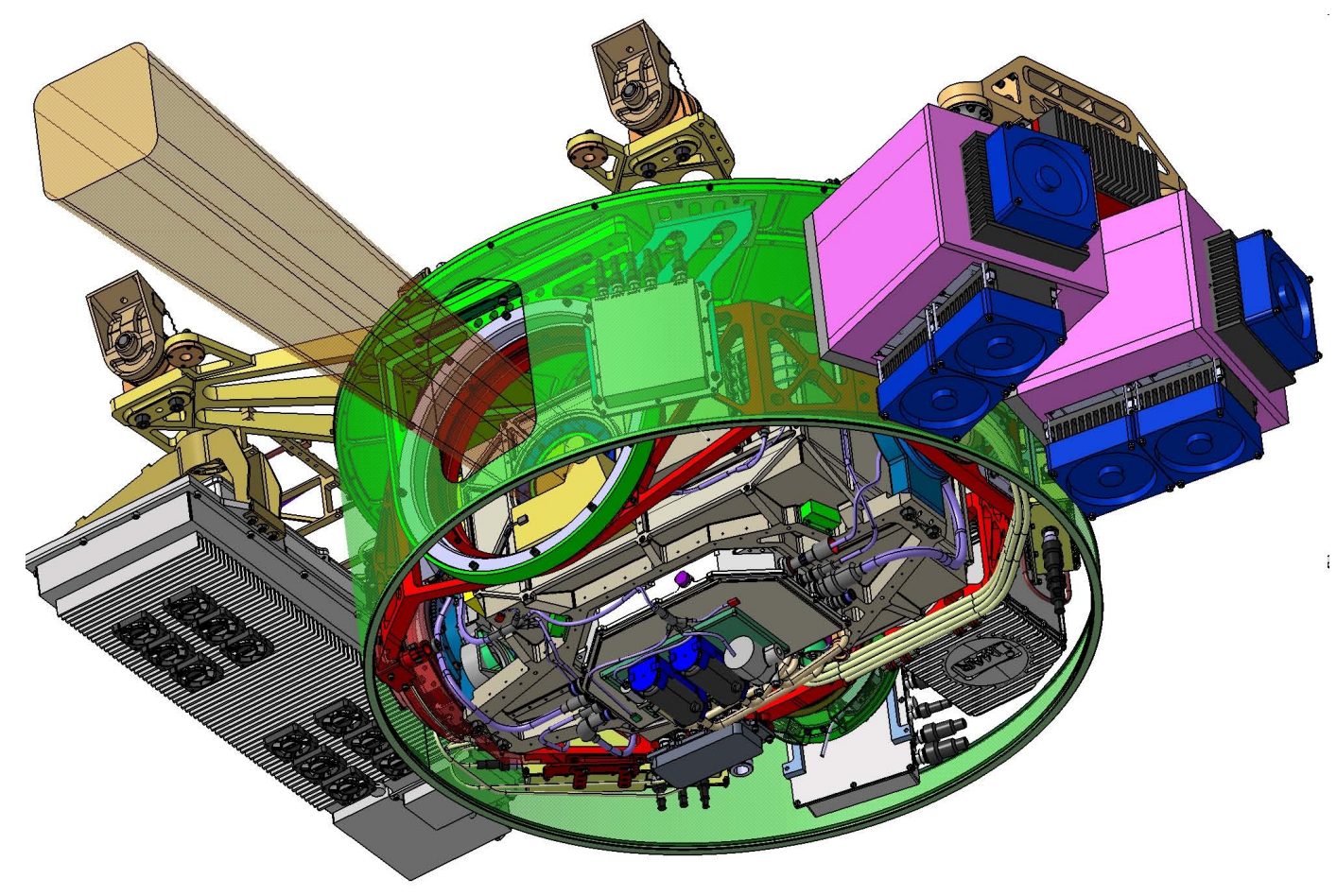

Fig. 1. Design of the GLORIA instrument. GLORIA will be housed in the belly pod of HALO and mounted on a base plate suspended from the aircraft. The picture shows a view where the carrier is omitted. The yellow beam indicates the viewing beam of the instrument. The GLORIA interferometer will be mounted in a cardanic frame for compensation of aircraft movements. The outermost, vertical axis is given by the green cylinder which also closes the belly pod to the air stream.

ratio, individual array pixels will be co-added to obtain sampling patterns of specific scientific measurement modes.

The GLORIA instrument has the ability to pan the instrument LOS relative to the flight direction. This is realized using a gimbal mount that also stabilizes the LOS during measurements. While conventional air-borne instruments usually point $90^{\circ}$ relative to the flight direction, GLORIA is capable of adjusting its LOS between $45^{\circ}$ and $135^{\circ}$ (as seen from above the carrier with $0^{\circ}$ being the flight direction of the carrier), which allows to view the same air volume from multiple angles. Depending on the aircraft, onto which GLORIA will be mounted, the possible range of angles may be slightly reduced due to obstructing parts of the aircraft. In combination with adapted flight paths of the carrier, this shall enable the retrieval of 3-D resolved air volumes.

Another advantage of the instrument concept is its great flexibility, i.e. the trade-off between spatial and spectral resolution can be adapted to the scientific needs. While currently only two modes are specified ("dynamics" and "chemistry" mode of Table 1), the hardware poses few restrictions should a certain scientific question require a different tradeoff between spatial and spectral sampling. As this paper explores the capabilities of GLORIA for tomographic retrievals, we focus on the measurement mode that offers the highest temporal sampling, which is the dynamics mode of
Table 1. As an example for the spectral resolution of the dynamics mode, simulated spectra for mid-latitude atmospheric conditions and $12 \mathrm{~km}$ tangent altitude are shown in Fig. 2. Even at this reduced spectral resolution, infrared emission features of important atmospheric constituents are clearly visible (e.g. $\mathrm{CO}_{2}, \mathrm{CFC} 11, \mathrm{CFC} 12, \mathrm{CCl}_{4}, \mathrm{H}_{2} \mathrm{O}, \mathrm{O}_{3}, \mathrm{HNO}_{3}$, $\mathrm{CH}_{4}, \mathrm{~N}_{2} \mathrm{O}$, or PAN, which Weigel et al., 2010 successfully retrieved using the CRISTA-NF instrument).

The focus of this paper is to describe a methodology that enables 3-D tomographic retrievals using simulated GLORIA measurements and to show the principal feasibility of this via simplified numerical experiments.

\section{A retrieval approach for large-scale inverse problems}

The inverse problem in the field of atmospheric limb sounding is to retrieve an atmospheric state, usually a vertical profile, from a set of emission spectra, usually taken from different tangent heights. Given an atmospheric state $\boldsymbol{x} \in \mathbb{R}^{n}$ it is possible to model the radiative transfer and the instrument effects with a model $\boldsymbol{F}$ to generate simulated measurements $\boldsymbol{y} \in \mathbb{R}^{m}$. $\boldsymbol{F}$ represents the actual physics as accurately as possible, given the constraints of understanding of physical processes and instrument effects, but also performance 


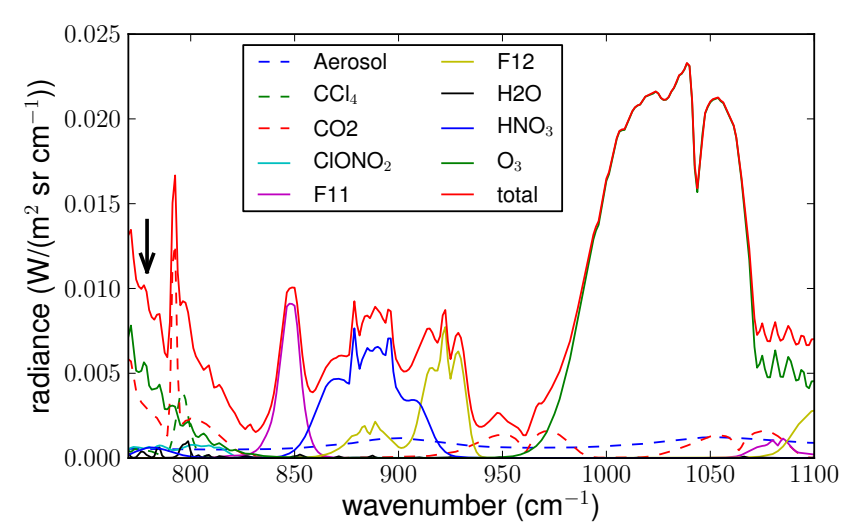

Fig. 2. Simulated radiance spectra for the GLORIA instrument operating in "dynamics" mode. Calculations are based on mid-latitude atmospheric conditions (Remedios et al., 2007) and $12 \mathrm{~km}$ tangent altitude. The black curve indicates total radiance. The other curves indicate radiance for single emitter atmospheres (see legend). The black arrow indicates the $\mathrm{O}_{3}$ line used for the retrievals.

constraints. The inverse problem then consists of deriving an atmospheric state from a set of noisy measurements, i.e.

$y=F(x)+\epsilon$

with $\epsilon \in \mathbb{R}^{m}$ representing the measurement errors. The current state-of-the-art in the field of atmospheric limb sounding is to measure and to retrieve 1-D profiles, possibly including gradients in the along-sight direction, or 2-D vertical planes accordingly (e.g. Roewe et al., 1982; Solomon et al., 1984; Livesey and Read, 2000; Carlotti et al., 2001; Steck et al., 2005; Carlotti et al., 2006). The general approach is independent of the dimensionality of the problem, but the computational complexity increases with the amount of involved unknowns and measurements.

GLORIA allows us to implement 3-D observation patterns in contrast to existing instruments. The associated 3-D tomographic reconstructions are extremely demanding on computational resources due to the large parameter space and the correspondingly large amount of measurements required to properly resolve the volume. Many different approaches have been tried to efficiently perform such a tomographic inversion, the most popular in the medical field being the filtered back-projection (e.g. Natterer, 2001). However, as the line of sights of GLORIA measurements do not span regular planes and due to the arbitrary shape of the carrier's flight path, a more flexible approach prevalent for resolving seismic measurements based on non-linear iterative minimization has been chosen (e.g. Rodgers, 2000; Nolet, 2008).

The inversion of $\boldsymbol{F}$ presents a non-linear, ill-posed, and in many cases both under- and over-constrained inversion problem. One way to obtain a unique and physically reasonable solution is regularization. We use a standard Tikhonov regularization approach as described, e.g. by Rodgers (2000). In our nomenclature, $\boldsymbol{x}_{\mathrm{a}} \in \mathbb{R}^{n}$ is the a priori knowledge about the state of the atmosphere, $\mathbf{S}_{\mathrm{a}} \in \mathbb{R}^{n \times n}$ is a covariance matrix for the a priori state $\boldsymbol{x}_{\mathbf{a}}, \boldsymbol{y} \in \mathbb{R}^{m}$ represents the vector of measurements and $\mathbf{S}_{\epsilon} \in \mathbb{R}^{m \times m}$ the covariance matrix of the measurement error. The method then computes a statistically optimal weighted average between the a priori information and the information contained in the measurements. The solution is calculated by minimizing the cost function $J: \mathbb{R}^{n} \mapsto \mathbb{R}$ with

$J(x)=\left(x-x_{\mathrm{a}}\right)^{T} \mathbf{S}_{\mathrm{a}}^{-1}\left(\boldsymbol{x}-\boldsymbol{x}_{\mathbf{a}}\right)$

$+(\boldsymbol{F}(\boldsymbol{x})-\boldsymbol{y})^{T} \mathbf{S}_{\epsilon}^{-1}(\boldsymbol{F}(\boldsymbol{x})-\boldsymbol{y})$.

A simple Newton iteration for solving the equation $J^{\prime}(\boldsymbol{x}) \stackrel{!}{=} 0$ gives the formula

$x_{i+1}=x_{i}-J^{\prime \prime}\left(x_{i}\right)^{-1} J^{\prime}\left(x_{i}\right)$

Approximating the Hessian matrix $J^{\prime \prime}\left(\boldsymbol{x}_{i}\right)$ by dropping all terms involving the second derivative of $\boldsymbol{F}$ (as they are too costly to calculate and hopefully negligible as the problem at hand is only "slightly" non-linear) results in the well-known Gauss-Newton iteration scheme

$\boldsymbol{x}_{i+1}=\boldsymbol{x}_{i}-\left(\mathbf{S}_{\mathrm{a}}^{-1}+\mathbf{F}^{\prime}\left(\boldsymbol{x}_{i}\right)^{T} \mathbf{S}_{\epsilon}^{-1} \mathbf{F}^{\prime}\left(\boldsymbol{x}_{i}\right)\right)^{-1}$
$\cdot\left(\mathbf{S}_{\mathrm{a}}^{-1}\left(\boldsymbol{x}_{i}-\boldsymbol{x}_{\mathrm{a}}\right)+\mathbf{F}^{\prime}\left(\boldsymbol{x}_{i}\right)^{T} \mathbf{S}_{\epsilon}^{-1}\left(\boldsymbol{F}\left(\boldsymbol{x}_{i}\right)-\boldsymbol{y}\right)\right)$,

where $\mathbf{F}^{\prime}\left(\boldsymbol{x}_{i}\right)$ is the Jacobian matrix of $\boldsymbol{F}$.

The matrix $\mathbf{S}_{\mathrm{a}}^{-1}$ is effectively chosen and defined by our regularization and can be considered as a tuning parameter (see Sect. 3.2). This method only delivers a maximum likelihood estimate if $\mathbf{S}_{\mathrm{a}}$ corresponds to the actual a priori covariance, which generally is not available or accurate enough (especially for 2-D and 3-D problems). However, the statistical interpretation of the matrices and the results remains valid as long as $\mathbf{S}_{\mathrm{a}}$ fulfills the formal requirements of a covariance matrix, i.e. being symmetric and positive semi-definite. The matrix $\mathbf{S}_{\epsilon}$ is assumed to be diagonal (or at least blockdiagonal), which means that $\mathbf{S}_{\epsilon}^{-1}$ has the same sparse structure and can be rather efficiently calculated.

In the following we will shortly present our forward model $\boldsymbol{F}$, describe how our regularization matrix $\mathbf{S}_{\mathrm{a}}^{-1}$ is set up and how to efficiently implement the inversion scheme for large tomographic problems.

\subsection{Fast forward model and Jacobian calculation}

For tomographic 3-D retrievals, the forward model needs to simulate many more measurements than is usual for a single conventional 1-D retrieval, so a fast forward model is of utmost importance.

A single measurement is generally simulated by casting multiple rays from the observer position, calculating the radiative transport along each ray and aggregating the resulting values according to a weighting function that is designed according to the field-of-view of the instrument. The reuse of a single ray for multiple measurements allows a significant reduction of computational effort for imager instruments. We therefore split the forward model $\boldsymbol{F}$ into a linear 
map $\mathbf{H} \in \mathbb{R}^{m \times p}$ and a non-linear function simulating the 1-D beams $\boldsymbol{P}: \mathbb{R}^{n} \mapsto \mathbb{R}^{p}$ with $\boldsymbol{F}(\boldsymbol{x})=\mathbf{H} \cdot \boldsymbol{P}(\boldsymbol{x})$. The matrix $\mathbf{H}$ is generally extremely sparse and stored accordingly.

The function $\boldsymbol{P}$ is realized using the emissivity growth approximation (EGA) method (e.g Weinreb and Neuendorffer, 1973; Gordley and Russell, 1981; Marshall et al., 1994). Our implementation of the EGA method was previously used as forward model for retrievals for several satellite- and airborne remote sensing experiments (e.g. Hoffmann et al., 2008, 2009; Eckermann et al., 2009; Hoffmann and Alexander, 2009). Compared with conventional line-by-line calculations this approach is about a factor 1000 faster, since the radiative transfer is calculated based on precalculated spectrally averaged values of emissivity stored in lookup tables. The emissivity look-up-tables for the forward model are prepared by means of exact line-by-line calculations utilizing the Reference Forward Model (RFM; Dudhia et al., 2002). Processing actual GLORIA measurements of the troposphere will likely require additional measures to reduce the error introduced by the approximate EGA method (e.g. Francis et al., 2006).

As seen in Eq. (4), the employed inversion algorithm requires the Jacobian matrix of $\boldsymbol{F}$ (also called the Kernel matrix). Given a fast enough forward model and exploiting the sparsity of the Jacobian matrix (Ungermann et al., 2010), it is feasible to determine the Jacobian matrix via finite differences even for tomographic problems of the given size. The Jacobian matrix $\mathbf{F}^{\prime}(\boldsymbol{x})$ for typical tomographic 3-D problems is very sparse as a single measurement is influenced only by a small fraction of the volume defined by $\boldsymbol{x}$. In our 3-D setups, generally about $99 \%$ of the Jacobian matrix entries are zero.

\subsection{Setup of regularization matrix}

For the regularization matrix $\mathbf{S}_{\mathrm{a}}^{-1}$, we use Tikhonov regularization matrices to assemble the inverse of a covariance matrix. This approach allows for an easy setup of $\mathbf{S}_{\mathrm{a}}^{-1}$, but not for $\mathbf{S}_{\mathrm{a}}$ itself. In effect, the variances of $\mathbf{S}_{\mathrm{a}}$ cannot be directly set opposed to approaches defining $\mathbf{S}_{\mathrm{a}}$ according to some adhoc distribution, e.g. a Gaussian distribution (e.g. Tarantola, 2004). However, inverting a dense $\mathbf{S}_{\mathrm{a}}$ directly or indirectly is computationally too costly, so we chose to define $\mathbf{S}_{\mathrm{a}}^{-1}$ instead.

We define four matrices $\mathbf{L}_{0}, \mathbf{L}_{1}^{x}, \mathbf{L}_{1}^{y}$, and $\mathbf{L}_{1}^{z} \in \mathbb{R}^{n \times n}$ corresponding to regularization of zero-th moment and first moment in the three dimensions. Then $\mathbf{S}_{\mathrm{a}}^{-1}$ is set to

$\mathbf{S}_{\mathrm{a}}^{-1}=\left(\left(\alpha_{0}\right)^{2} \mathbf{L}_{0}^{T} \mathbf{L}_{0}+\left(\alpha_{1}^{x}\right)^{2} \mathbf{L}_{1}^{x^{T}} \mathbf{L}_{1}^{x}\right.$

$\left.+\left(\alpha_{1}^{y}\right)^{2} \mathbf{L}_{1}^{y^{T}} \mathbf{L}_{1}^{y}+\left(\alpha_{1}^{z}\right)^{2} \mathbf{L}_{1}^{z^{T}} \mathbf{L}_{1}^{z}\right)$

with the tuning parameters $\alpha_{0}, \alpha_{1}^{x}, \alpha_{1}^{y}$, and $\alpha_{1}^{z} \in \mathbb{R}$.

The matrix $\mathbf{S}_{\mathrm{a}}^{-1}$ becomes singular if $\alpha_{0}$ is set to zero, so the Gauss-Newton iteration defined in Eq. (4) should be replaced by a Levenberg-Marquardt iteration scheme to ensure convergence (with more care in parameter choice the scheme presented in Appendix B should also be applicable).

The matrix $\mathbf{L}_{0}$ is simply the inverse of a diagonal matrix containing the a priori standard deviations. The $\mathbf{L}_{1}$ matrices are simple Tikhonov regularization matrices of first order, adjusted for the non-uniform grid size (see Appendix A for a detailed discussion). This implies that $\alpha_{0}$ is unit-less and $\alpha_{1}$ has the unit of length divided by the unit of the retrieval target type. Together, the matrices penalize deviations of the retrieved profile from the a priori profile and of the three partial derivatives of the retrieved profile in the direction of the three spatial dimensions from the corresponding partial derivative of the a priori profile.

\subsection{Iterative techniques for solving large-scale linear equation systems}

The iteration formula in Eq. (4) is ideal for tomographic retrievals, as all involved matrices are sparse matrices. This significantly reduces both memory consumption and computation time.

The matrix inversion seemingly implied in Eq. (4) is naturally not performed naively, but a linear equation system is solved instead. Using Cholesky- or QR-decompositions becomes quickly infeasible for tomographic problems, as it either consumes prohibitive amounts of computation time or becomes numerically unstable so that it requires special treatment.

The linear equation system in each step is defined by the matrix $\mathbf{C} \in \mathbb{R}^{n \times n}$ :

$\mathbf{C}=\left(\mathbf{S}_{\mathrm{a}}^{-1}+\mathbf{F}^{\prime}\left(\boldsymbol{x}_{i}\right)^{T} \mathbf{S}_{\epsilon}^{-1} \mathbf{F}^{\prime}\left(\boldsymbol{x}_{i}\right)\right)$.

For our choice of $\mathbf{S}_{\mathrm{a}}^{-1}$, this matrix is symmetric positive definite suggesting an iterative conjugate gradient scheme. As $\mathbf{C}$ is most likely not very sparse due to the term $\mathbf{F}^{\prime}\left(\boldsymbol{x}_{i}\right)^{T} \mathbf{S}_{\epsilon}^{-1} \mathbf{F}^{\prime}\left(\boldsymbol{x}_{i}\right)$, we avoid calculating it altogether, as the generation itself is quite costly and consumes a large amount of memory. Fortuitously, the conjugate gradient algorithm does not require the matrix $\mathbf{C}$, but only the result of products of $\mathbf{C}$ with given vectors. This product can be cheaply provided by four matrix-vector products, i.e. multiplying the given vector first with $\mathbf{F}^{\prime}\left(\boldsymbol{x}_{\boldsymbol{i}}\right)$, then the result of this product with $\mathbf{S}_{\epsilon}^{-1}$ and so on.

Equation (4) also lends itself well to algorithmic differentiation methods that do not calculate the full Jacobian matrix, but offer a way to efficiently calculate the product of the Jacobian matrix, its transpose or even the Hessian matrix with a given vector.

As we found the convergence speed of the conjugate gradient method lacking for our 3-D tomographic setups (especially for multi-target setups involving targets of different magnitudes), we employed a simple Jacobi preconditioning scheme (e.g. Saad, 2003). Most pre-conditioners require full knowledge of the matrix $\mathbf{C}$. For the Jacobi preconditioning only the diagonal of $\mathbf{C}$ is required, which can be calculated 
using only about $O(\mathrm{~nm})$ multiplications if $\mathbf{S}_{\epsilon}^{-1}$ is a diagonal matrix. If the non-diagonal elements of $\mathbf{S}_{\epsilon}^{-1}$ are not zero and cannot be neglected, this preconditioning requires an additional multiplication of the Jacobian with $\mathbf{S}_{\epsilon}^{-1}$, i.e. $O\left(\mathrm{n} \mathrm{m}^{2}\right)$ multiplications neglecting the sparsity of the involved matrices.

The conjugate gradient scheme requires a convergence criterion to determine if the current solution is good enough. We terminate the conjugate gradient scheme, as soon as the residuum has been reduced by a certain percentage. We found the Gauss-Newton scheme to converge faster, if for the first iterations only a small reduction of the residuum is required, tightening the convergence criterion as the GaussNewton iteration converged upon the solution. In addition to the outer Gauss-Newton iteration converging faster, this naturally also decreases the number of inner iterations used in the conjugate gradient scheme (see Appendix B).

\subsection{Memory-conserving diagnostics}

We determine the retrieval diagnostics according to Rodgers (2000). This generally requires the calculation of the gain matrix $\mathbf{G}$ that maps differences of measurements onto differences of retrieval result and the averaging kernel matrix $\mathbf{A}$ that maps the true atmospheric state onto the retrieval result. With $\hat{\boldsymbol{x}}$ being the retrieval result, the gain matrix is defined as

$\mathbf{G}=\left(\mathbf{S}_{\mathrm{a}}^{-1}+\mathbf{F}^{\prime}(\hat{\boldsymbol{x}})^{T} \mathbf{S}_{\epsilon}^{-1} \mathbf{F}^{\prime}(\hat{\boldsymbol{x}})\right)^{-1} \mathbf{F}^{\prime}(\hat{\boldsymbol{x}})^{T} \mathbf{S}_{\epsilon}^{-1}$

and the averaging kernel matrix (also called resolution matrix in the field of seismology) is defined as

$$
\begin{aligned}
& \mathbf{A}=\mathbf{G} \mathbf{F}^{\prime}(\hat{\boldsymbol{x}})=\left(\mathbf{S}_{\mathrm{a}}^{-1}+\mathbf{F}^{\prime}(\hat{\boldsymbol{x}})^{T} \mathbf{S}_{\epsilon}^{-1} \mathbf{F}^{\prime}(\hat{\boldsymbol{x}})\right)^{-1} \\
& \cdot \mathbf{F}^{\prime}(\hat{\boldsymbol{x}})^{T} \mathbf{S}_{\epsilon}^{-1} \mathbf{F}^{\prime}(\hat{\boldsymbol{x}}) .
\end{aligned}
$$

With $\boldsymbol{x}_{\mathrm{t}}$ being the true state of the atmosphere and $\boldsymbol{\epsilon}$ again the measurement errors, the retrieval result can be expressed by the linear expression

$\hat{\boldsymbol{x}}=\mathbf{A} \boldsymbol{x}_{\mathrm{t}}+(\mathbf{I}-\mathbf{A}) \boldsymbol{x}_{\mathrm{a}}+\mathbf{G} \epsilon$,

which gives the basis for the (linear) diagnostics.

The gain matrix $\mathbf{G}$ can then be used to estimate the influence of measurement noise on the retrieval result: $\mathbf{S}_{\text {noise }}=\mathbf{G S}_{\epsilon} \mathbf{G}^{T}$. The averaging kernel matrix can be used to deduce various interesting quantities, including measurement contribution and resolution (e.g. Rodgers, 2000; von Clarmann et al., 2009).

For large 2-D or 3-D setups, the full matrix-inversion in Eqs. (7) and (8) is computationally too costly and furthermore numerically unstable. Further, even if the matrices could be reliably generated, they become too large to keep fully in memory. For example, for our simple baseline setup (see Sect. 4.1), the full averaging kernel matrix alone would require about 80 Gigabyte of memory in double precision.
It is however possible to derive diagnostics for a single point without any matrix inversion as first demonstrated by Nolet (1985). Applying this approach onto Rodgers' more advanced diagnostics, for the diagnostics of a single point only the corresponding row of $\mathbf{G}$ is needed to estimate the noise error (which is generally estimated using the corresponding diagonal element of $\mathbf{S}_{\text {noise }}$ ) and only the corresponding row of $\mathbf{A}$ is needed to derive measurement contribution and resolution.

To derive a single row of $\mathbf{A}$ and $\mathbf{G}$, only a single row of

$\mathbf{C}=\left(\mathbf{S}_{\mathrm{a}}^{-1}+\mathbf{F}^{\prime}(\hat{\boldsymbol{x}})^{T} \mathbf{S}_{\epsilon}^{-1} \mathbf{F}^{\prime}(\hat{\boldsymbol{x}})\right)^{-1}$

is needed, as this row vector can then be multiplied using vector-matrix multiplications from the left to the right with $\mathbf{F}^{\prime}(\hat{\boldsymbol{x}})^{T} \mathbf{S}_{\epsilon}^{-1}$, respectively $\mathbf{F}^{\prime}(\hat{\boldsymbol{x}})^{T} \mathbf{S}_{\epsilon}^{-1} \mathbf{F}^{\prime}(\hat{\boldsymbol{x}})$ to derive the rows of $\mathbf{G}$ and $\mathbf{A}$.

As $\mathbf{C}^{-1}$ is symmetrical, so is $\mathbf{C}$. This simplifies the problem, as columns of $\mathbf{C}$ can be easily calculated using the same iterative linear equation system solver described in the preceding section, and using the proper unit vector for the righthand-side.

Estimating the covariance of the smoothing error requires an actual covariance matrix describing the actual statistics of the atmosphere, which our ad-hoc covariance matrix cannot deliver. However, assuming that the employed covariance matrix were the true one, the smoothing error

$\mathbf{S}_{\mathrm{s}}=(\mathbf{A}-\mathbf{I}) \mathbf{S}_{\mathrm{a}}(\mathbf{A}-\mathbf{I})^{T}$

can also be computed for individual data points. The $i$-th element of the diagonal of $\mathbf{S}_{\mathrm{s}}$ is the $i$-th row of $(\mathbf{A}-\mathbf{I})$ multiplied with $\mathbf{S}_{\mathrm{a}}$ multiplied with the $i$-th column of $(\mathbf{A}-\mathbf{I})^{T}$ (being naturally identical with the $i$-th row of $(\mathbf{A}-\mathbf{I}))$. The corresponding row of $\mathbf{A}$ was already previously calculated and subtracting one for the subtraction of $\mathbf{I}$ is trivial. The matrixvector product with $\mathbf{S}_{\mathrm{a}}$ can be replaced by another application of the conjugate gradient scheme using $\mathbf{S}_{\mathrm{a}}^{-1}$ as equation system and the modified row of $\mathbf{A}$ as right-hand-side.

Using this method, it is possible to retrieve diagnostic information for individual points of $\boldsymbol{x}$ without keeping the full matrices $\mathbf{G}$ or $\mathbf{A}$ in memory or even fully generating them. For tomographic retrievals, it is too costly to determine the diagnostics for all points of the retrieval as this generally requires far more computation time than the retrieval itself. But for our tomographic setups, we found the diagnostic quantities to generally vary only slowly from point to point, so that one can use Monte-Carlo techniques to randomly sample a subset of $\boldsymbol{x}$ to get quickly a good impression of the overall quality and characteristics of the retrieval and to determine the areas that deserve a closer look. 


\subsection{Discrete representation of the atmosphere}

Large-scale, 3-D inversions require an efficient representation of the atmospheric state. Temperature, pressure, aerosol and a varying number of trace gases need to be represented with sufficient detail over the whole volume from which emitted infrared radiance can reach the detector of the instrument. A simple extension of the typical one-dimensional discretization with linear interpolation for the representation of profiles has been used for the results in this paper. A cuboid of atmosphere is discretized by sampling it at the cross-points of a rectilinear grid. Between the grid points, the value is linearly interpolated between the eight corners of the cube. The grid needs not be fully regular and thereby allows for a denser representation in the area with the most measurements (in our setup the center) and a sparser representation at other places.

\section{Numerical experiments}

This section describes several numerical experiments performed for the GLORIA instrument. Their purpose is on the one hand to demonstrate the feasibility of large-scale 3-D tomographic inversions using the methodology presented in Sect. 3, on the other hand to explore the tomographic capabilities of the GLORIA instrument itself.

\subsection{Simulation setup}

One intent of the simulations is to demonstrate the feasibility of tomographic retrievals. To that end, we employ a 3-D GEM-AQ (Global Environmental Multi-scale model, Air Quality; Kaminski et al., 2008) data set as our test atmosphere, which is used to generate simulated measurements of GLORIA. The general idea is to reconstruct the original 3-D atmosphere from simulated measurements. To keep the inversion rather simple, only a single target shall be reconstructed. We chose ozone instead of the simpler and perhaps more natural temperature as it can be used as a tracer to examine the UTLS air exchange and therefore fits to the scientific focus of GLORIA. According to GEM-AQ model simulations of the synoptic situation during the European heat wave of July 2006, ozone also exhibits quite interesting horizontal structures close to a tropopause fold (Struzewska and Kaminski, 2008). Figure 3 shows part of one such structure, an ozone filament over the north west of France at $200 \mathrm{hPa}$ with a horizontal extension of about $250 \mathrm{~km} \times 2000 \mathrm{~km}$. This filament is a remnant of upper stratospheric air mixed into the lower-most stratosphere. Ozone values inside this filament are about $50 \%$ increased in comparison to the ambient air.

The horizontal sampling of the GEM-AQ data set is about $12 \mathrm{~km}$ while the vertical resolution in the UTLS region is about $1 \mathrm{~km}$. For our simulation we picked a volume from

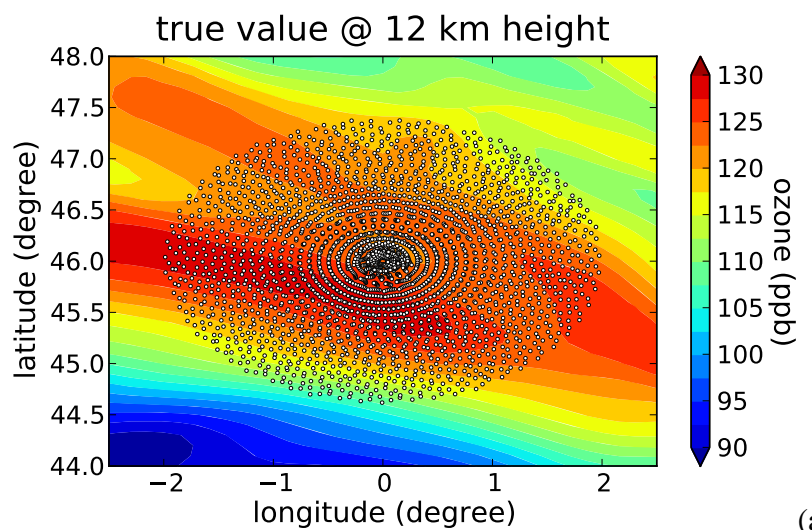

(a)

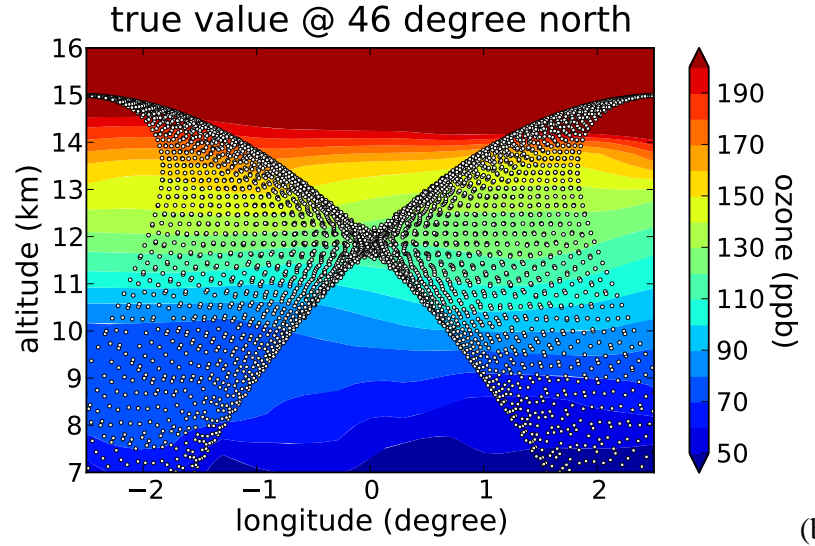

(b)

Fig. 3. Two cuts through the full 3-D true atmospheric state. (a) Shows the ozone filament at $12 \mathrm{~km}$ height, while (b) shows a vertical cut along $46^{\circ} \mathrm{N}$. Tangent points close to the cut planes are shown as small white circles.

$8.64^{\circ} \mathrm{E}$ to $8.64^{\circ} \mathrm{W}$ and from $40.00^{\circ} \mathrm{N}$ to $52.00^{\circ} \mathrm{N}$. For generating the simulated measurements, this region is sampled with a horizontal grid size of $\approx 11 \mathrm{~km}$. For the inversion, the atmosphere is sampled with this fine grid only from $2.88^{\circ} \mathrm{E}$ to $2.88^{\circ} \mathrm{W}$ and from $44.00^{\circ} \mathrm{N}$ to $48^{\circ} \mathrm{N}$. The outer region is covered by only three samples on each side to reduce the amount of unknowns. The vertical sampling is $1 \mathrm{~km}$ from $1 \mathrm{~km}$ to $6 \mathrm{~km}$ altitude, $0.25 \mathrm{~km}$ from $6 \mathrm{~km}$ to $16 \mathrm{~km}, 1 \mathrm{~km}$ from $16 \mathrm{~km}$ to $20 \mathrm{~km}$, and $2 \mathrm{~km}$ from $20 \mathrm{~km}$ to $60 \mathrm{~km}$. The retrieval shall derive ozone in the vertical region from $4 \mathrm{~km}$ to $20 \mathrm{~km}$ over the full horizontal extent from the simulated measurements. The inversion is simplified by assuming perfect knowledge of the top-column above $20 \mathrm{~km}$ and all entities except ozone in the retrieved area. In this idealized study, we concentrate on the properties of the tomographic retrieval method and therefore neglect other uncertainties. The simulated measurements are generated by the same radiative transfer model that is also used in the retrieval.

We use temperature, pressure, $\mathrm{CCl}_{4}, \mathrm{CO}_{2}, \mathrm{ClONO}_{2}, \mathrm{H}_{2} \mathrm{O}$, $\mathrm{HNO}_{3}$ and ozone in our simulations as these contribute most of the radiation around $778.5 \mathrm{~cm}^{-1}$. As not all these trace gases are contained in the GEM-AQ model data, we take $\mathrm{CCl}_{4}, \mathrm{CO}_{2}, \mathrm{ClONO}_{2}$, and $\mathrm{HNO}_{3}$ from mid-latitude profiles 
of Remedios et al. (2007). For this study, we assume clearsky conditions.

Taking the envisioned amount of measurements for the presented tomographic retrieval will take about $90 \mathrm{~min}$, as the speed of the carrier is limited. During that time, the state of the atmosphere may change significantly due to advection or chemical reactions. Handling this will require special treatment, which is beyond the scope of this paper. Therefore, we assume an unchanging atmosphere during the time required to take the measurements.

The employed regularization scheme uses the ozone midlatitude values and standard deviations of Remedios et al. (2007) for $\boldsymbol{x}_{\mathrm{a}}$ and $\mathbf{L}_{0}$. As initial guess $\boldsymbol{x}_{0}$, we take ozone values for polar conditions from the same source. The parameter $\alpha_{0}$ has been set to 0.1 to reduce the bias towards the a priori profile. The retrievals seem not to require this term, but we added it to ensure that $\mathbf{S}_{\mathrm{a}}^{-1}$ is a regular, well-conditioned matrix. As it is also symmetric and positive definite, it is invertible and its inverse can be interpreted as a covariance matrix. The parameter $\alpha_{1}^{z}$ has been set to $4 \times 10^{-4} \mathrm{~km} / \mathrm{ppb}$ and $\alpha_{1}^{x}$ and $\alpha_{1}^{y}$ both to $0.8 \mathrm{~km} / \mathrm{ppb}$. In contrast to the zero-th moment term $\alpha_{0}$ the smoothing constraints are required for the well-behavior of the retrieval. One can loosely interpret the $\alpha_{1}$ parameters as "correlation length" divided by the product of the square root of 2 times a standard deviation (Steck and von Clarmann, 2001). Using the standard deviation of ozone at $12 \mathrm{~km}$ from Remedios et al. (2007) as a basis, this corresponds roughly to a vertical correlation length of $\approx 0.3 \mathrm{~km}$ and a horizontal correlation length of $\approx 600 \mathrm{~km}$. A parameter study showed that these overall and relative weights deliver the visually best results (that is the results were not too "noisy" on the one hand, but also not too smoothened on the other hand). Further, varying the overall regularization strength, i.e. scaling all $\alpha$ 's with a factor, and plotting the L-curve, i.e. the deviation of the measurements against the constraint divided by the scaling factor (e.g. Hansen and O'Leary, 1993) shows that the regularization strength is Lcurve optimal, i.e. it represents a good balance between fidelity to the measurements and smoothing due to the constraint. The retrieval results are robust against small changes of the tuning parameters. Reducing the horizontal tuning parameters below, e.g. $2 \times 10^{8} \mathrm{~km}$ leads to noticeable noisiness and increasing it over $16 \times 10^{8} \mathrm{~km}$ to noticeable smoothing (both not depicted). The vertical smoothing seems not to be essential and in fact, the presented retrievals converge to similar results without vertical smoothing. It has however a noticeable effect on the retrieval result above the instrument altitude of $15 \mathrm{~km}$, where insufficient measurement information is available and the regularization parameter has been chosen as the smallest value, for which acceptable results in that height range are retrieved. The resulting system matrix $\mathbf{C}$ has a condition number of approximately 450000 , which allows for a reasonably quick convergence of the conjugate gradient scheme.
The results presented here use measurements which are each averaged over two rows of the GLORIA detector, i.e. over 256 detector pixels and thereby deviate slightly from the envisioned co-adding of the "dynamics" mode. As the coadding is performed off-line, this requires no change to the instrument whatsoever. We average over two rows instead of a single one largely because our atmosphere model does not offer such fine vertical details in the region of interest and we could significantly reduce simulation time by this simplification. In the presence of clouds, affected detector pixels would be removed from the measurement. The measurements are generated by our radiative transfer model, which is also used for the retrieval. The measurement noise is modelled by a stochastic additive offset component and a stochastic multiplicative gain component. This assumes that any systematic component can be handled by calibration or during the retrieval itself. According to the specification of GLORIA, the offset component for 256 averaged pixels is expected to have a standard deviation of $0.1875 \times 10^{-5} \mathrm{~W} /\left(\mathrm{m}^{2} \mathrm{sr} \mathrm{cm}^{-1}\right)$. For the gain component for 256 averaged pixels, we assume that a stochastic component with a standard deviation of $0.1 \%$ remains. We perform both simulations with and without added noise according to this specification as presented below. The simulations use only a single channel at $778.5 \mathrm{~cm}^{-1}$, which was previously used by Weigel et al. (2010) to derive ozone from CRISTA-NF measurements. This channel is optically thin in the troposphere, close to the $\mathrm{CO}_{2}$ Q-branch at $12.6 \mu \mathrm{m}$ usually employed for deriving temperature, and relatively unaffected by water vapor. A realistic setup would naturally need more channels to compensate for instrument effects, aerosol, etc. On the other hand, at least for $\mathrm{O}_{3}$ it is possible to add further channels with strong $\mathrm{O}_{3}$ contribution to reduce measurement noise. A single image with the described 64 individual measurements will be generated by GLORIA every $\approx 3 \mathrm{~s}$. For the description of the employed panning, see Sect. 4.3.

Retrieving this baseline setup involves 6 iterations and uses roughly $60 \mathrm{CPU} \mathrm{h}$, respectively $9 \mathrm{~h}$ of real time on an eight-core machine consuming only about $1.3 \mathrm{~Gb}$ of memory total. Thereby about $90 \%$ of the computation time is used for calculating the Jacobian matrix once in the beginning and once for the final step and diagnostics. We found that intermediate updates of the Jacobian matrix were of no benefit for the setup at hand.

\subsection{Analysis of baseline setup}

We first present and analyze the baseline setup in greater detail. The aircraft is assumed to fly a circle with $\approx 400 \mathrm{~km}$ diameter over the north of France at $15 \mathrm{~km}$ height as is depicted in Fig. 4. Within this circle, at $12 \mathrm{~km}$ height, an ozone filament is clearly visible (shown in Fig. 3), which is the "target" of the retrieval. Within this paper, the tangent points of measurements close to the cut plane are usually drawn as small white circles. Their presence indicates the part of 


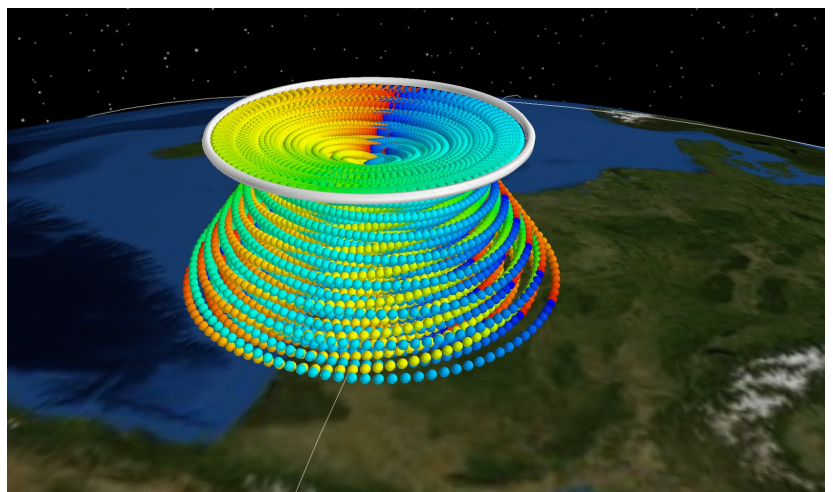

Fig. 4. A circular flight path including the location of the tangent points of the measurements as color-coded spheres (red being the first measurements, blue the last ones) and the flight-track as a white tube.

the volume, where good agreement between the retrieved atmospheric state and the true atmospheric state is expected. Please note the fundamental difference between the tangent point distribution in Fig. 4 and what is usually found for 2-D tomographic retrievals from satellite borne limb-sounder data. Where the latter have an even distribution of tangent points nearly over the whole 2-D plane, the tangent points in the presented 3-D setup are clustered in the middle of the volume. In the 3-D setup, most line-of-sights of measurements pass largely through atmosphere outside the region covered with tangent points, especially those measurements for lower altitude. This makes the presented 3-D tomographic retrieval much more influenced by boundary effects and overall more ill-posed and dependent on a-priori constraints than current 2-D tomographic retrievals.

The diagnostic information for the baseline setup was derived with the technique described in Sect. 3.4. Only the depicted planes were calculated and required several times as much computation time as the retrievals themselves (one depicted plane including all diagnostic entities costs $\approx 250 \mathrm{CPU}$ ). Figure 5 shows the retrieval result for the baseline setup with added instrument noise. Figure 5a shows that the filament is qualitatively well reproduced and Fig. 5b indicates that the error in the region covered with tangent points is well below $\pm 5 \%$. The vertical cuts in Fig. $5 \mathrm{c}$ and $\mathrm{d}$ show good agreement at other heights.

The influence of noise on the retrieval result can be well seen by comparing Fig. 5 with Fig. 6, where the same setup is simulated without instrument noise. The deviations from the true value outside of the central region are still present, which indicates that these are not an effect of noise, but of insufficient measurement information. Further simulations (not depicted) indicate that decreasing the noise by means of averaging over time does not improve the result as fewer measurements deliver less spatial resolution.
The given simulation setup has a high measurement contribution close to one within the convex region spanned by the tangent points (not depicted) indicating a negligible bias of the retrieval result towards the a priori vector $\boldsymbol{x}_{\mathrm{a}}$. The influence of instrument noise at $12 \mathrm{~km}$ height is generally less than $\approx 3$ ppb in the region covered by tangent points.

The resolution is determined by calculating the FWHM of the corresponding row of the averaging kernel matrix in the three dimensions. This is naturally only a meaningful measure if the volume, where the row of the averaging kernel matrix is larger than half its maximum, has an approximately ellipsoid shape, being nicely aligned with the major axis. We found this to be the case within the region covered by tangent points. Outside this region, it may also be, e.g. of a "banana" shape, so the resolution cannot be easily expressed with just three numbers. That aside, the vertical resolution is $\approx 325 \mathrm{~m}$ in the region covered by tangent points at $12 \mathrm{~km}$ height as is shown in Fig. 7. The achievable vertical resolution peaks here, as at $12 \mathrm{~km}$ height the best horizontal coverage with tangent points is given. Simulations with a finer height grid of $125 \mathrm{~m}$ and 128 distinct vertical measurements per image (not depicted) indicate that a vertical resolution of $\approx 200 \mathrm{~m}$ seems possible. In Fig. $7 \mathrm{~b}$ one can see that the achievable vertical resolution in the "tangent point holes" at the bottom and at the top of the analyzed volume increases rapidly due to a lack of measurements. This suggests that the radius of the circle, respectively the height of the aircraft, which determine the height region with best tangent point coverage, should be chosen according to the height region one is most interested in.

The horizontal resolution in longitudinal direction in Fig. $8 \mathrm{a}$ is around $20 \mathrm{~km}$ to $30 \mathrm{~km}$ indicating that the chosen sampling grid is fine enough to resolve all information present. The low values at the upper and lower part of Fig. 8a result from the fact that the shape of the rows of the averaging kernel matrix in this region deteriorates from a roughly ellipsoid shape towards something resembling more "bananas", which extend roughly in north-south direction. Correspondingly, the horizontal resolution in latitudinal direction is quite similar to that in longitudinal direction within the region covered by tangent points, but increases rapidly towards the north and south and is deceivingly low towards the east and west. Figure $8 \mathrm{~b}$ indicates that the horizontal resolution is best in the middle of the volume, where the air volume is seen from most angles. Towards the border, especially to the lower left and lower right, the coverage decreases and therewith also the horizontal resolution. By tuning the regularization parameters (not depicted), it is possible to shift information between vertical resolution, horizontal resolution and measurement noise. We found the current setup sufficient for our purposes and reserve a thorough analysis of the regularization scheme. 


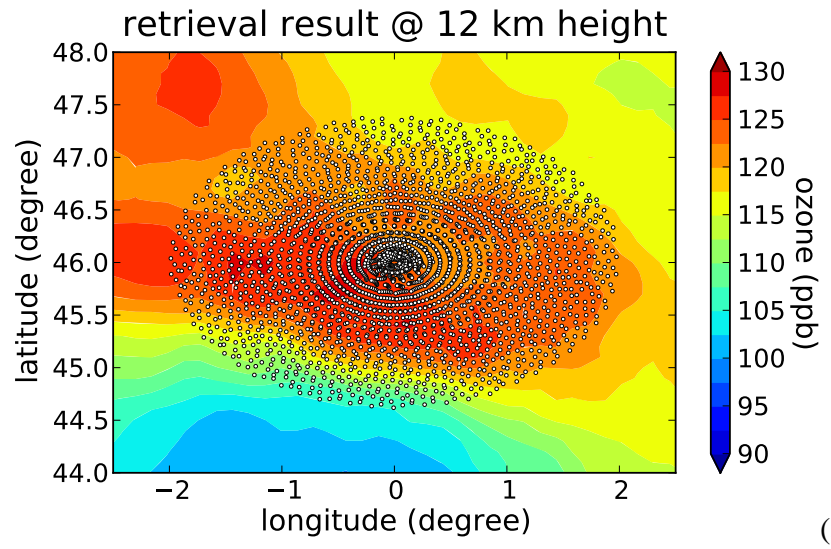

(a)

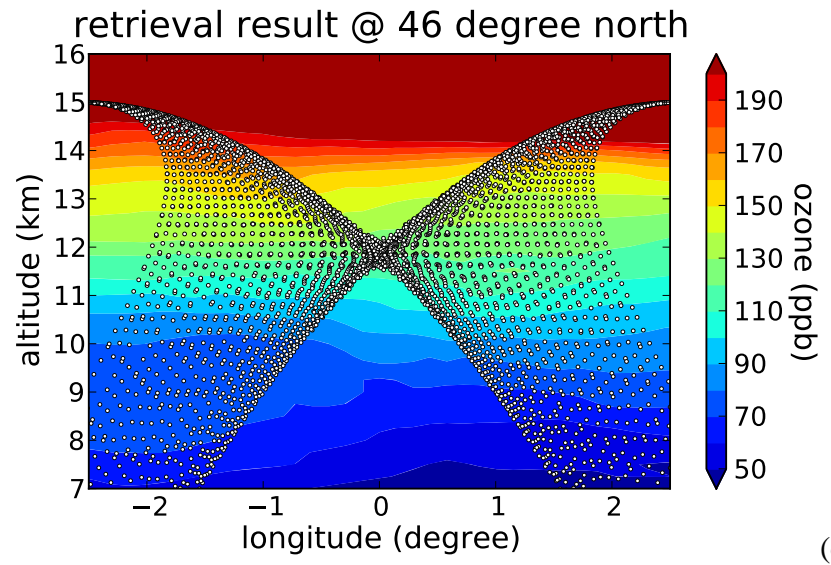

(c)

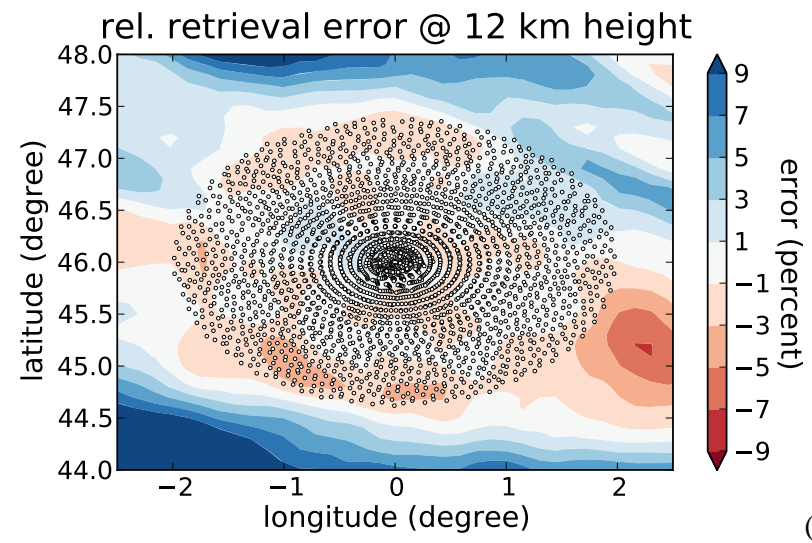

(b)

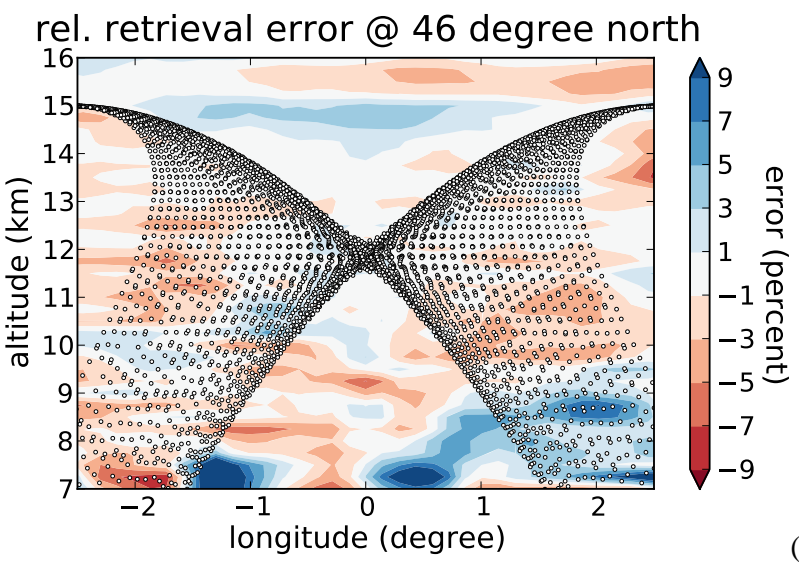

(d)

Fig. 5. The retrieval result of the baseline setup with added instrument noise. (a) Shows the retrieved ozone values at $12 \mathrm{~km}$ height while (b) depicts the relative error in percent compared to the true values. (c) and (d) Shows the same information for a vertical cut at $46^{\circ} \mathrm{N}$.

\subsection{Effect of panning}

An innovative new feature of the GLORIA instrument is the ability to point the detector in different horizontal directions without changing the flight direction of the aircraft itself to enable 3-D tomographic reconstructions.

We examined the effect of panning on the tomographic reconstruction to determine, in which way this feature actually benefits the retrieval result. The baseline setup described in Sect. 4.2 made use of the full GLORIA panning capability by moving the detector by $4^{\circ}$ between taking two images and going back to $45^{\circ}$ as soon as an angle of $135^{\circ}$ is reached. Figure 9 shows an exemplary retrieval without any panning, i.e. fixing the instrument viewing direction to $90^{\circ}$ like a conventional instrument would measure. The strength of the regularization has not been changed by, e.g. increasing the horizontal regularization strength to retain comparability of the results. As can be seen in Fig. 9a, the tangent points of measurements do not span a volume but are located on a 2-D surface in space. Consequently, the retrieval does not deliver a good representation of the true atmosphere, which we were unable to significantly improve by, e.g. tuning the regularization. Figure $9 \mathrm{~b}$ depicts the retrieved atmospheric state. It seems difficult to discern the original filament from this data as the relative error exceeds $-10 \%$ even close to the tangent points in the middle.

Better results can be achieved with a reduced panning in contrast to no panning at all. Figure 10 depicts a retrieval setup, in which the panning is reduced to the range from $65^{\circ}$ to $115^{\circ}$ relative to the flight direction, but the filament is still difficult to discern. Our simulations indicate that the results improve the wider the angle gets, with an optimum close to the angle available for GLORIA. Extending the fan further (not depicted), more rays pass through air volumes that are not viewed from multiple angles and are therefore difficult to resolve three-dimensionally. In addition, these rays are missing on the inside of the flight path, reducing the retrieval quality there.

A second consideration must be given to the panning step size, in which the available panning angle is used. While the simulations did not show the result being very sensitive to it, it has been found that the result improves the more evenly distributed the tangent points of the individual measurements are. Reducing the step size increases the number of tangent 


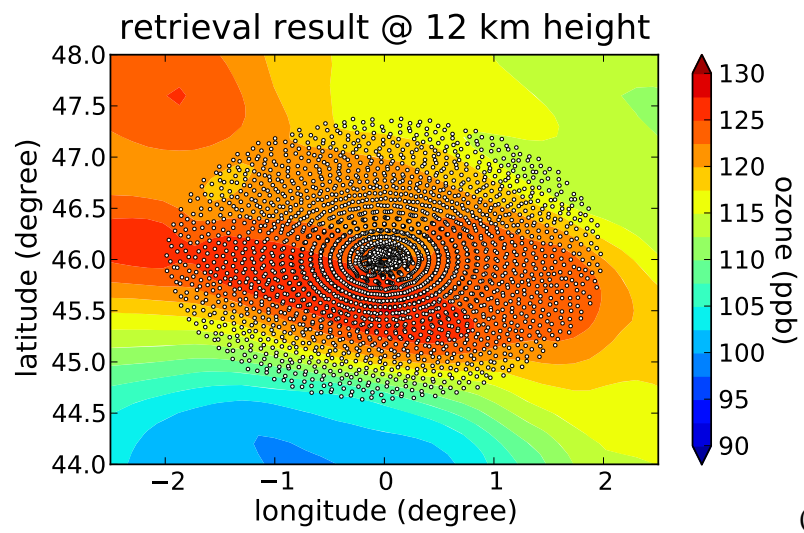

(a)

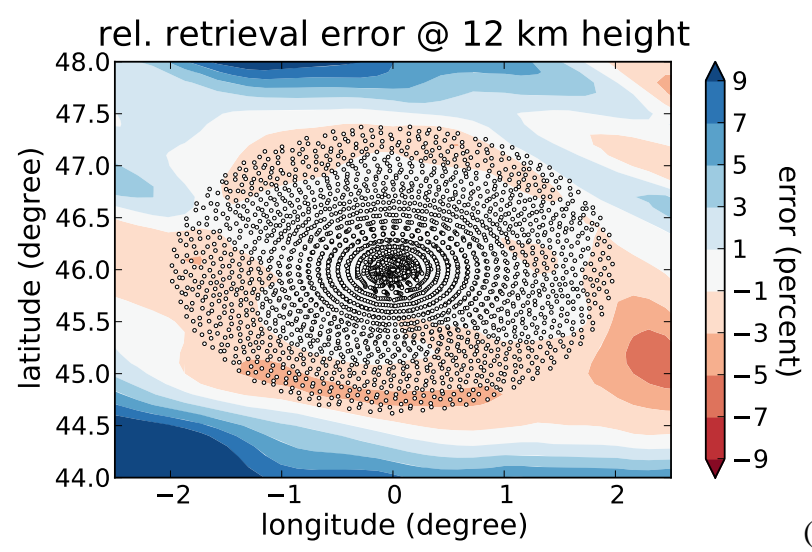

(b)

Fig. 6. The retrieval result of the baseline setup without added instrument noise. (a) Shows the retrieved ozone values at $12 \mathrm{~km}$ height while (b) depicts the relative error in percent compared to the true values.

point "circles", but reduces the number of points on the "circle". For the "dynamics mode", we found the natural step size of $4^{\circ}$ optimal for our setup, while, e.g. for the "chemistry mode", which requires longer time for a single measurement, a larger step size might be chosen.

In summary, the ability to pan the instrument is clearly an invaluable asset for tomographic reconstruction.

\subsection{Choice and effect of flightpath}

The choice of flightpath is of large importance for 3-D tomographic retrievals. Closed or nearly closed paths seem to be able to provide a good reconstruction of the true atmospheric state, similar to computer tomography. In our experiments the true atmospheric state was only reproduced accurately in those parts of the volume, where the tangent points of measurements are located. In effect, the flightpath and panning (see Sect. 4.3) should be chosen accordingly. A perfectly circular flight path gives the most regular distribution of tangent points as presented in Sect. 4.1. As such a flight path may not be feasible for practical concerns, we will discuss in

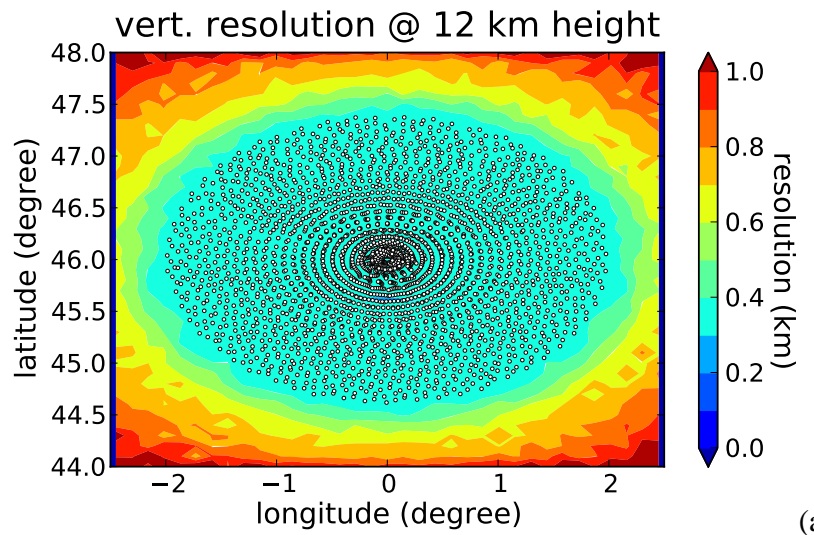

(a)

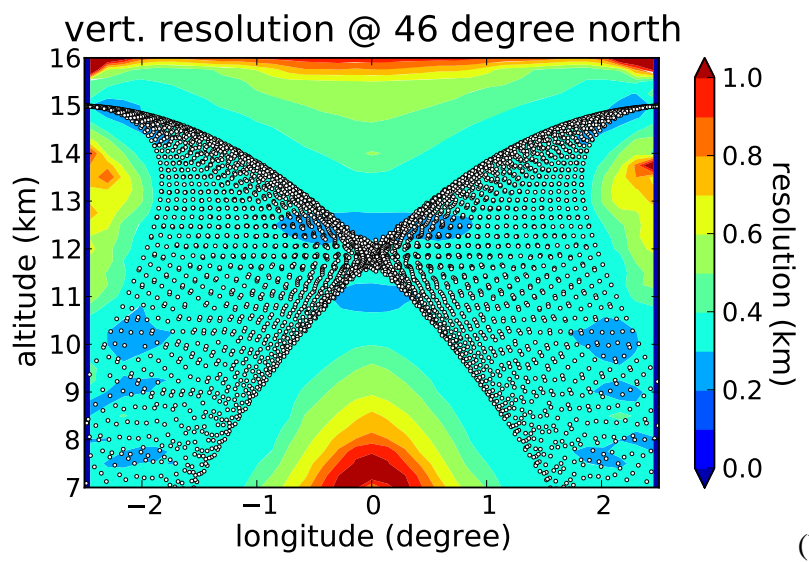

(b)

Fig. 7. The vertical resolution of the baseline setup. (a) Shows the vertical resolution at $12 \mathrm{~km}$ altitude while (b) depicts the vertical resolution at $46^{\circ} \mathrm{N}$.

the following how approximations to the ideal circular track behave.

We present two such approximations, one being a square flightpath and one being an octagonal flight path. Figure 11 depicts a simple square track in the same style as Fig. 4. One can see that the area below the corners of the square is devoid of measurements while there are large extensions below the sides of the square (the latter ones could be avoided by influencing the panning in a more complicated way than employed here). Figure $11 \mathrm{~b}$ shows the relative error of the retrieval result for this flight path at $12 \mathrm{~km}$ height. One can see good agreement where tangent points are located, but the corners - being devoid of tangent points - are already quite erroneous. Calculating horizontal resolution (not depicted) indicates that the values in the corners are nearly purely derived by horizontal smoothing. Figure 12 shows in comparison an octagonal flight path, which resembles a circular track more closely. Figure 12a depicts again the location of tangent points in space and time and Fig. $12 \mathrm{~b}$ shows the relative error of the retrieval result at $12 \mathrm{~km}$ height. The area covered by tangent points is larger, corresponding with a larger area of 


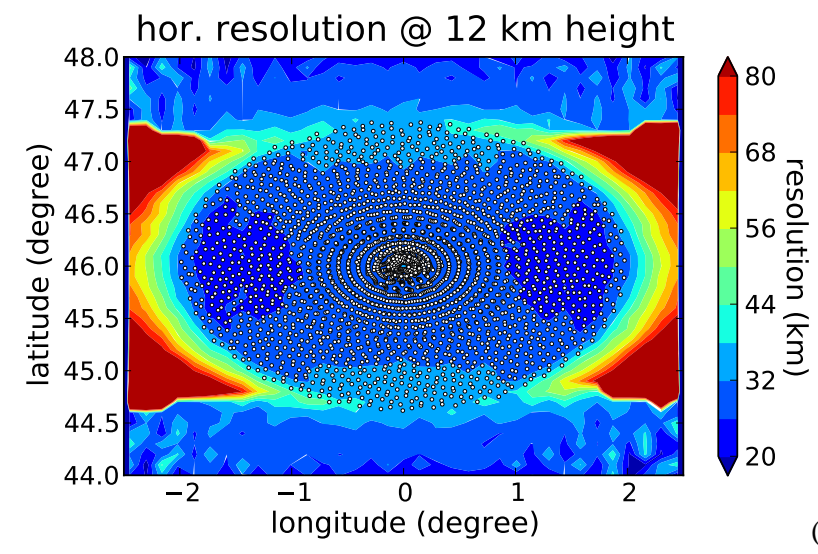

(a)

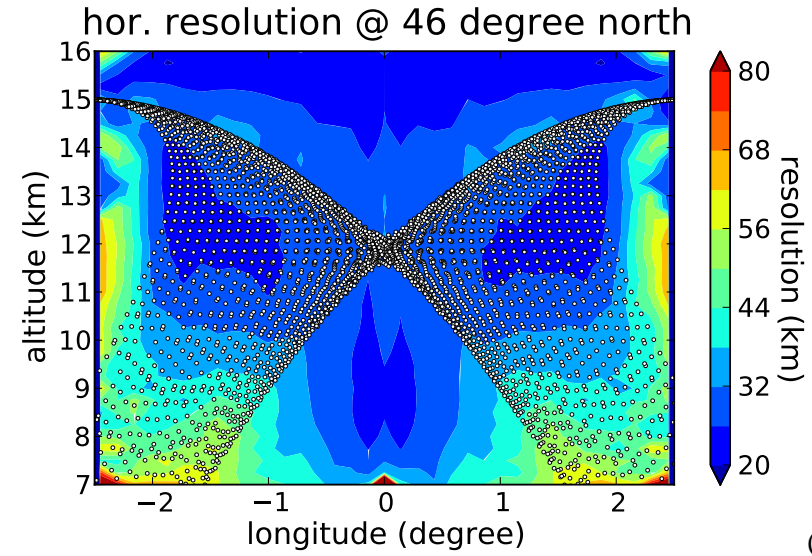

(b)

Fig. 8. The horizontal resolution in longitudinal direction of the baseline setup. (a) Shows the horizontal resolution at $12 \mathrm{~km}$ altitude while (b) depicts the horizontal resolution at $46^{\circ} \mathrm{N}$.

good agreement. In principle, this seems to be a good compromise as a larger area is covered by tangent points so that the true ozone structure can actually be identified better than in Fig. 3a. However the horizontal resolution is generally higher than for the baseline setup and the vertical resolution quickly becomes larger in between the "petals".

Both presented approximations to a circular flight track seem usable and could possibly be optimized further by adjusting, e.g. the panning. We seem to be able to trade horizontal resolution for covered volume, so the flight path can be determined according to scientific need. Therefore, it is expected that a workable flight track can be found for campaigns given typical restrictions imposed by aerospace regulations and scientific goals.

\subsection{Comparison with individual 1-D retrievals}

The 3-D tomographic retrieval is more robust in the presence of horizontal inhomogeneity than conventional 1-D retrievals. This section demonstrates that the employed test case is indeed a difficult one for conventional retrievals due to the horizontal inhomogeneity of ozone and develops a
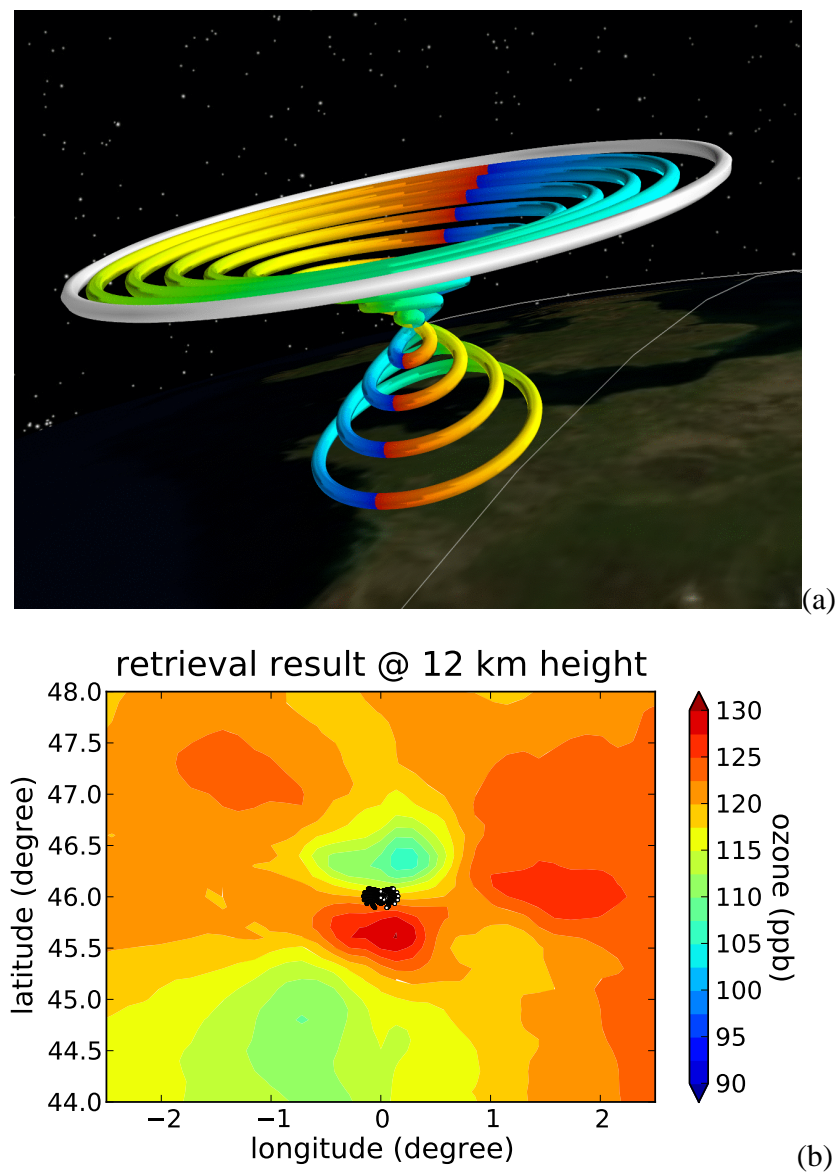

(b)

Fig. 9. This figure demonstrates a tomographic retrieval without panning. (a) Shows the location of the tangents of the measurements as color-coded spheres (red being the first measurements, blue the last ones) and the flight-track as white tube. (b) Shows the retrieval result at $12 \mathrm{~km}$ height.

method to quantify the effect of horizontal inhomogeneity on 1-D retrievals based upon the assumption of horizontal homogeneity.

To compare the tomographic approach with conventional retrievals, each individual image of the standard setup was used to perform a single 1-D retrieval. To exclude secondary representation effects, only ozone within the retrieval height range was kept three-dimensionally varying; every other quantity was made horizontally homogeneous. It was found that the 1-D retrievals require a stronger vertical regularization (i.e. $\alpha_{1}^{z}=4 \times 10^{6}$ ) as the regularizing influence of the horizontal smoothing is missing. Figure 13 depicts as an example the results of the 1-D retrievals at $12 \mathrm{~km}$ height with the true atmosphere as background. One sees clearly that ozone is severely underestimated depending on the pointing of the measurement instrument. Profiles underestimating ozone are generally derived from images pointing northwards and profiles overestimating it are generally pointing southwards. Increasing the strength of the vertical smoothing 


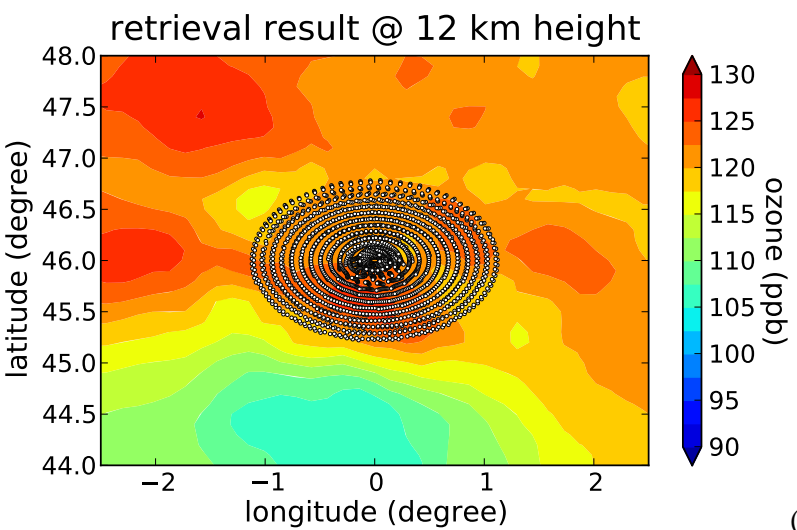

(a)

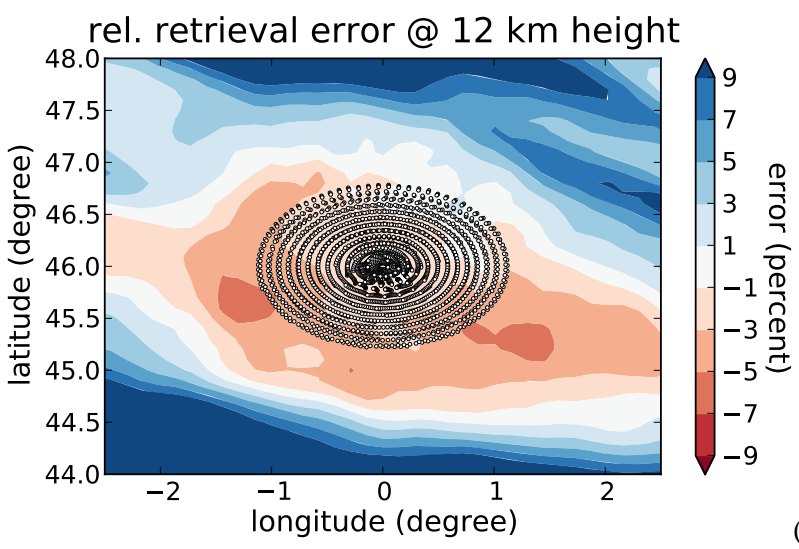

(b)

Fig. 10. Depicted is the retrieval result (a) and relative error (b) of a tomographic reconstruction with panning being reduced to a span of $50^{\circ}$ instead of $90^{\circ}$.

further, the noisiness of the results decreases at the cost of a worsening vertical resolution. However, the systematic under- and overestimation is not affected by a stronger or weaker regularization.

To gain deeper insight into the cause of these systematic errors, a single retrieval with an underestimation of $\approx 20 \%$ was selected for analysis, which is indicated by a broad black circle in Fig. 13. Figure 14 shows the rays of the corresponding image together with the ozone values along the line-of-sights. To compensate for the strong vertical gradient of ozone, the values have been horizontally normalized to the ozone concentration at the line defined by the tangent point respectively the horizontal instrument location above $15 \mathrm{~km}$. Above $10 \mathrm{~km}$ altitude, one can see a strong horizontal structure with ozone concentrations decreasing away from the instrument. This gradient certainly violates the assumption of horizontal homogeneity. Figure 15b shows the row of the 1-D averaging kernel matrix corresponding to the value at $12 \mathrm{~km}$. One sees the expected vertical averaging, but this is not the reason for the underestimation as the ozone concentrations at, above, and below $12 \mathrm{~km}$ are quite similar. No indication of a problem is given by this 1-D diagnostic.
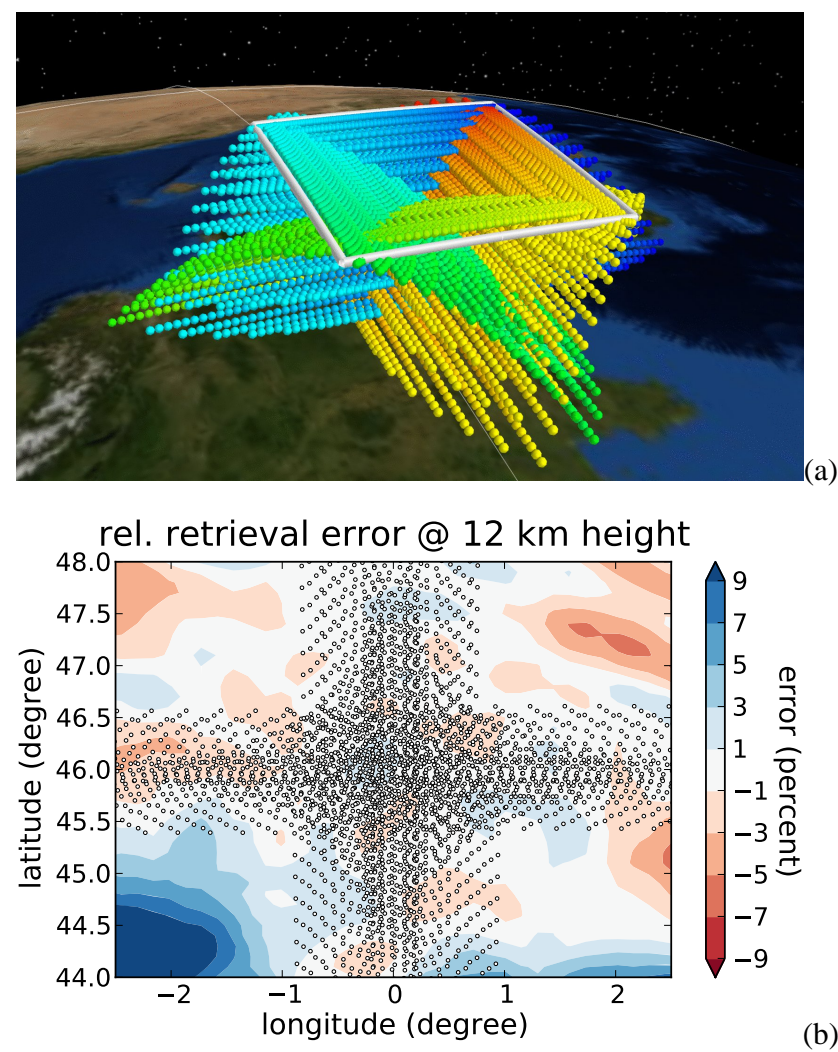

(b)

Fig. 11. This figure demonstrates a square flight path. (a) Shows the location of the tangents of the measurements as color-coded spheres (red being the first measurements, blue the last ones) and the flighttrack as white tube. (b) Shows the relative error of the retrieval result at $12 \mathrm{~km}$ height.

As the usual 1-D diagnostics depend on the notion of a horizontally homogeneous atmosphere, they cannot capture the effects of 2-D structures. Instead, a mapping of the true in this case -2-D atmosphere onto the 1-D results is required to gain further insight into the problem. Such a map can be generated by using the 1-D gain matrix shown in Fig. 15a and a 2-D Jacobian matrix describing the sensitivity of the measurements to a 2-D atmosphere. A 2-D Jacobian can simply be generated by creating a 2-D representation of the atmosphere and filling it with the true state of the atmosphere along this cut (it can be alternatively filled with the horizontally homogeneous retrieval result, if the true state is unavailable). This 2-D atmosphere employed by us uses the same altitude grid as the 1-D representation and uses a regular horizontal grid with a $5 \mathrm{~km}$ horizontal spacing. Now, the 1-D gain matrix can be multiplied with the 2-D Jacobian matrix to create a new averaging kernel matrix that maps the true 2-D state of the atmosphere to the 1-D retrieval result. This is quite similar to the way that the usual averaging kernel matrix is calculated, the only difference being the exchange of the 1-D Jacobian matrix by the 2-D Jacobian matrix. The resulting matrix is referred to as 2-D/1-D averaging kernel 

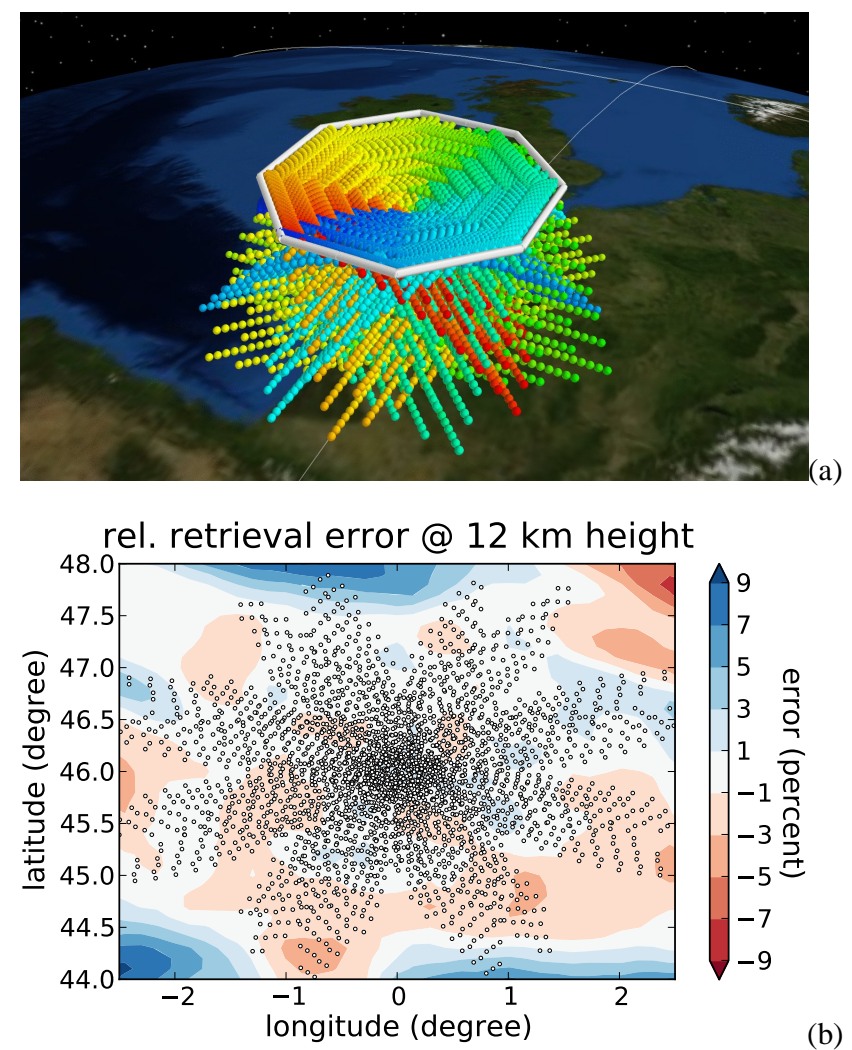

(b)

Fig. 12. This figure demonstrates an octagonal flight path. (a) Shows the location of the tangents of the measurements as colorcoded spheres (red being the first measurements, blue the last ones) and the flight-track as white tube. (b) Shows the relative error of the retrieval result at $12 \mathrm{~km}$ height.

matrix and shown in Fig. 16. A similar technique was used by von Clarmann et al. (2009) to estimate the horizontal resolution of the MIPAS instrument.

The 2-D/1-D averaging kernel matrix is closely related to the usual 1-D averaging kernel matrix. For instance, the 2-D/1-D averaging kernel matrix can be transformed to the 1-D averaging kernel matrix by summing up all elements of each row belonging to the same altitude. Consequently, the horizontal oscillations above $12 \mathrm{~km}$ in Fig. 16 sum up horizontally to (nearly) zero. If there were a horizontally constant ozone concentration at higher altitudes, the oscillations would not affect the retrieval result, just as the 1-D averaging kernel matrix suggests. However, there is a strong gradient, which is folded with the oscillations visible in the 2-D/1-D averaging kernel matrix and thereby contributes noticeably to the retrieval result at $12 \mathrm{~km}$ altitude.

The observed horizontal oscillations in the 2-D/1-D averaging kernel matrix are the consequence of the averaging kernel matrix $\mathbf{A}$ being a linear combination $\left(\mathbf{A}=\mathbf{G F}^{\prime}(\hat{\boldsymbol{x}})\right)$ of the rows of the Jacobian matrix, i.e. the weighting functions. Figure 15a shows a row of the gain matrix; it depicts the coefficients used to add up the rows of the Jacobian matrix to

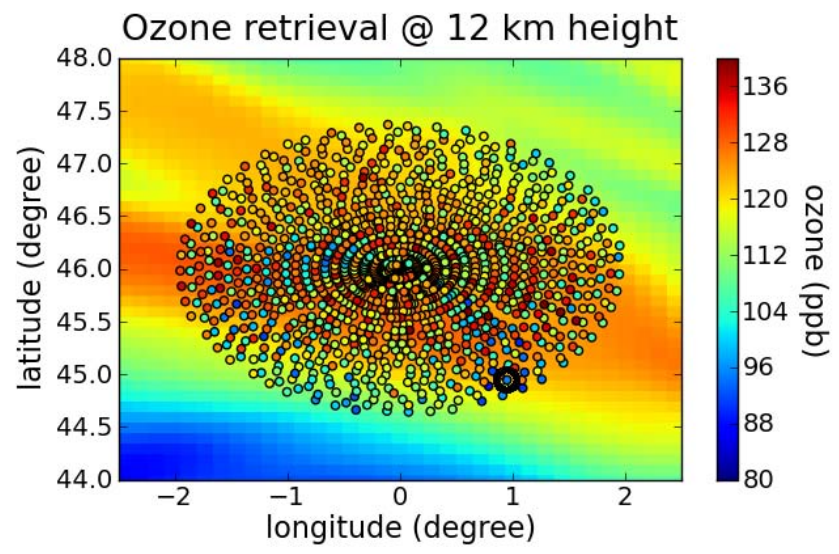

Fig. 13. The retrieval results for $12 \mathrm{~km}$ height of all 1-D retrievals shown as color-coded circles placed at the horizontal location of the closest tangent point. The data point highlighted by a broad black circle has been selected for closer examination. The background shows the true state of the atmosphere.

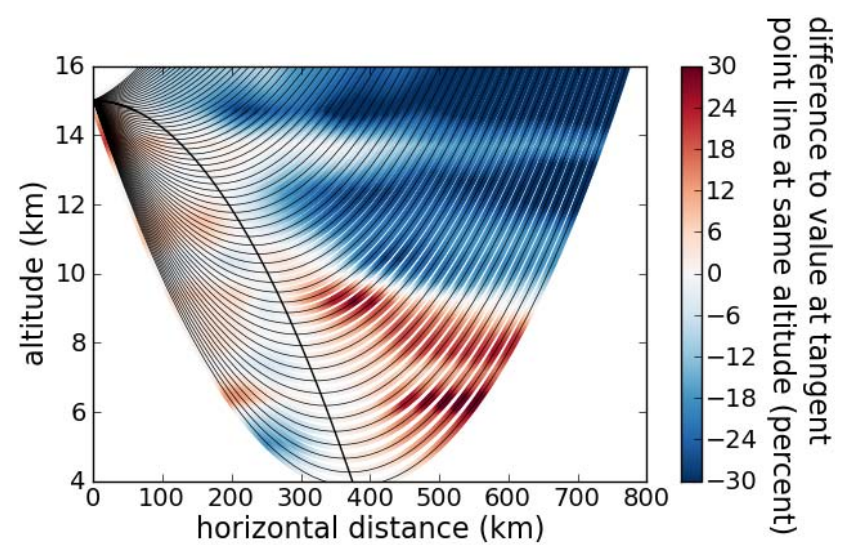

Fig. 14. Depicted are the rays of an exemplary 1-D retrieval. The ozone values are depicted relative to the value at the line of tangent points. The tangent points are shown as bold line starting from the instrument to the left and arcing down towards the ground.

generate the row of the averaging kernel matrix for $12 \mathrm{~km}$ altitude. It is foremost a weighted average of the measurements with tangent points close to $12 \mathrm{~km}$. Figure 16a shows one row of the 2-D Jacobian matrix, i.e. a 2-D weighting function describing the sensitivity of one measurement with tangent point altitude close to $12 \mathrm{~km}$ altitude to changes of the 2-D atmosphere. Its maximum sensitivity lies close to the tangent point, but significant contributions are also present at higher altitudes. The negative entries in Fig. 15a are there to remove these contributions in the 1-D averaging kernel matrix. Ignoring the horizontal dimension, the 1-D weighting functions can be used to very closely approximate the delta function as shown in Fig. 15b. However, if the 2-D Jacobian matrix is employed, it becomes apparent that the available measurements do not in fact allow such a good spatial 

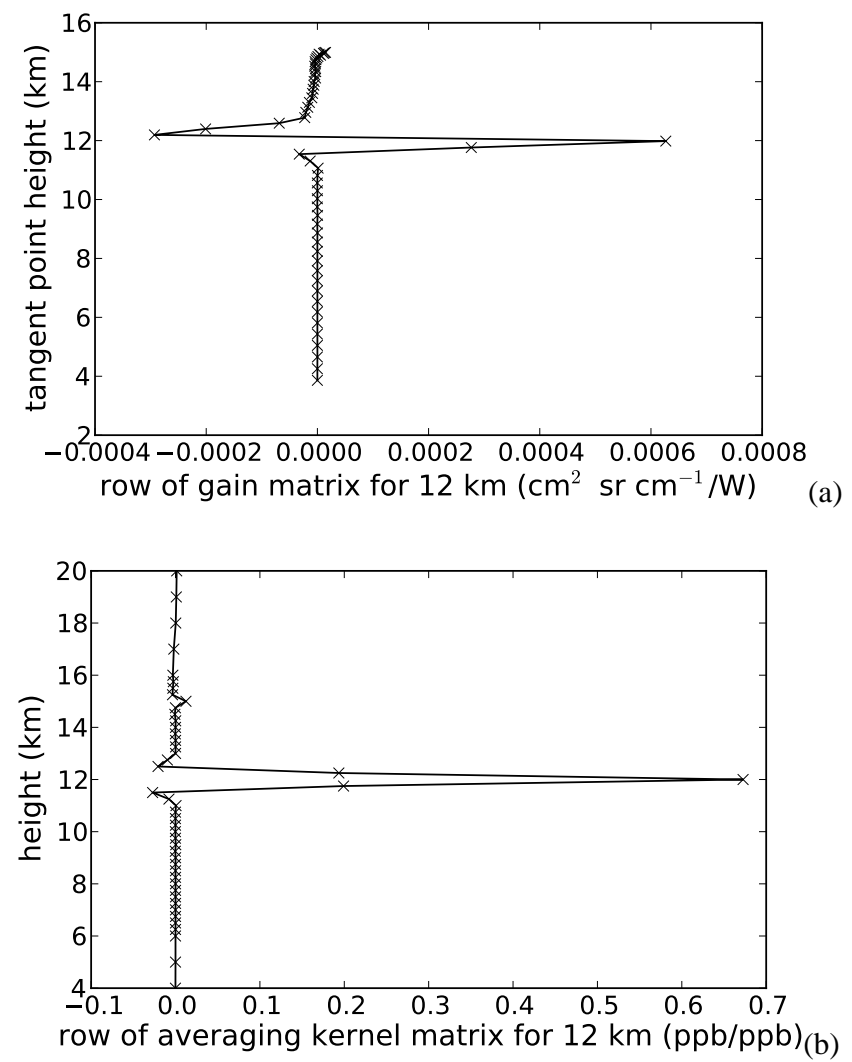

Fig. 15. (a) Shows the row of the gain matrix corresponding to the retrieval result at $12 \mathrm{~km}$. (b) Shows the row of the 1-D averaging kernel matrix describing the relationship between the true atmosphere and the 1-D retrieval result at $12 \mathrm{~km}$.

localization. Only by adding additional measurements viewing the volume from multiple angles an averaging kernel matrix really resembling a delta peak can be constructed.

It is now clear, what causes the $\approx 20 \%$ underestimation in the case at hand. The horizontal oscillations of the 2-D/1-D averaging kernel matrix in combination with the decreasing ozone concentrations away from the instrument cause this underestimation. The effect is especially pronounced as ozone concentrations increase with height and therefore small oscillations in the 2-D averaging kernel far above the retrieved value still contribute meaningfully. For trace gas species that remain approximately constant or decrease in concentration with altitude the effect should be less pronounced due to the decrease in air pressure with height. The same problem has affected the evaluation of MIPAS measurements as demonstrated by Kiefer et al. (2010). Naturally, the oscillations of the 2-D averaging kernel matrix at higher altitudes contribute only noticeably to the result, if the atmosphere is not in fact horizontally homogeneous.

In contrast, the 3-D retrieval is able to use more measurements and thereby more weighting functions in the construction of the averaging kernel matrix. This increases the vector

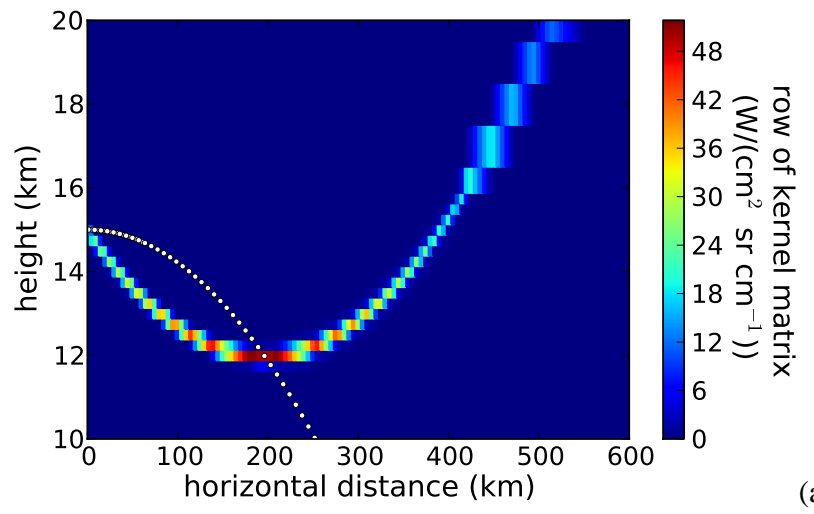

(a)

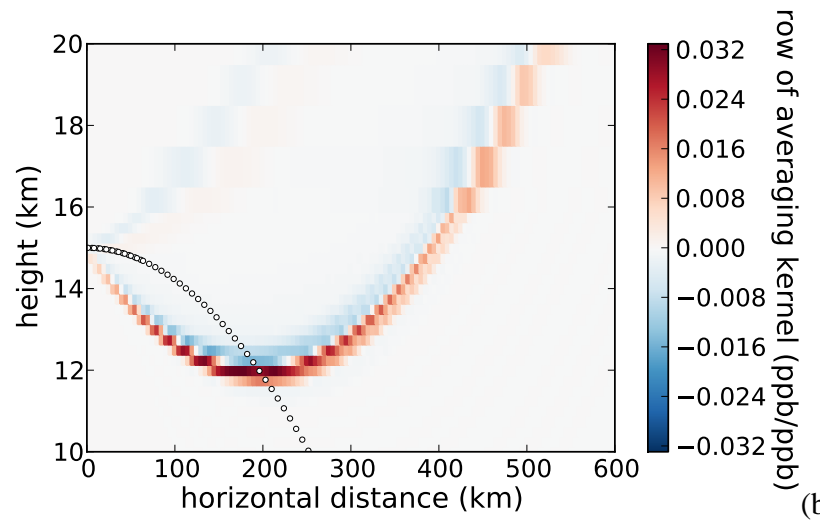

(b)

Fig. 16. This figure examines the effect of the assumption of horizontal homogeneity on a single 1-D retrieval more closely. (a) Shows exemplarily the weighting function with tangent height closest to $12 \mathrm{~km}$. The 1-D weighting function corresponds to the horizontal sum of this matrix. (b) Depicts the row associated with $12 \mathrm{~km}$ retrieval height of the full 2-D averaging kernel matrix that shows the contributions of higher layers to the retrieval result.

space spanned by the weighting functions and thereby offers better approximations to the desired delta functions.

The described technique can be used to more reliably compare 3-D model data with retrieved 1-D profiles by folding the model data with the 2-D/1-D averaging kernel matrix instead of the usual 1-D averaging kernel matrix. The method can also be used to quantify the effect of the horizontal inhomogeneity on 1-D retrieval results and thereby in combination with a chemical weather forecast help identify problematic atmospheric situations and accordingly optimize flightpaths of airborne instruments to avoid a bias in the retrieval results. 


\section{Conclusions}

We introduced a methodology allowing for efficient tomographic retrievals from limb measurements that can be applied to both satellite- and air-borne instruments. We demonstrated how the use of sparse matrices combined with iterative solvers deliver in an efficient manner a solution to the non-linear, ill-posed problem of tomography. We also showed how the result of such an inversion can be analyzed using the powerful diagnostic tools of Rodgers (2000) without generating the dense gain and averaging kernel matrices for all data points. The presented numerical inversion scheme is able to efficiently handle the massive amount of measurements and unknowns of tomographic retrievals and is both able to handle the described 3-D setups, but is also well suited for 2-D retrievals of satellite measurements.

We employed this method on simulated measurements of the GLORIA instrument to demonstrate its capability to three-dimensionally resolve air volumes. The new GLORIA instrument combines an infrared 2-D detector array with a Michelson interferometer mounted in a highly flexible gimbal and is unique in its combination of very high spatial and state of the art spectral resolution and its ability to scan the line-of-sight (LOS) $90^{\circ}$ horizontally. This is a significant advance compared to previous limb sounders, which are limited by their restricted measurement geometry. An ozone filament at $12 \mathrm{~km}$ found in GEM-AQ simulations served as a test setup.

We found that using nearly circular flightpaths, the 3-D structure of the ozone filament could be qualitatively well reproduced in the region well covered by tangent points of measurements. As a perfectly circular flight track is probably infeasible, we examined different closed curves and found that also approximations to a circle deliver very useful results. A 3-D resolution of $\approx 325 \mathrm{~m}$ vertically and of $\approx 25 \mathrm{~km} \times 25 \mathrm{~km}$ horizontally was achieved in our test retrievals. The vertical resolution can likely be improved to $200 \mathrm{~m}$ with a different setup.

One of the innovative features of the GLORIA instrument is its ability to horizontally pan the LOS of the instrument away from the typical angle of $90^{\circ}$ relative to the flight direction of its carrier. It was demonstrated that this feature is a key component that enables the tomographic retrieval capability.

Finally, we set up a series of conventional 1-D retrievals based on the assumption of horizontal homogeneity. We found that these retrievals were subject to large systematic errors depending on the direction of the LOS. We used a 2-D/1-D averaging kernel matrix to analyze the 1-D retrieval and found that the 2-D averaging kernel matrix is subject to horizontal oscillations, which affect the retrieval result in the presence of strong gradients and high concentrations in upper layers. This affects especially the retrieval of ozone but should also be considered for other species. A tomographic retrieval can use more measurements from different angles to subdue these oscillations and derive a better inversion result.

The GLORIA instrument should be able to derive highly resolved 3-D volumes of the UTLS enabling a better understanding of meso-scale processes and structures in this region. The combination of the 2-D detector array and the ability to pan the LOS are able to deliver sufficient measurements from different angle to achieve a 3-D reconstruction with vertical resolution of $\approx 200 \mathrm{~m}$ and horizontal resolution of $\approx 25 \mathrm{~km}$. This should allow GLORIA to deliver results with a spatial resolution that is unprecedented for current limb sounders.

\section{Appendix A}

\section{Setup of $L_{1}^{z}$}

The three-dimensional, rectilinear grid is defined by three vectors $\boldsymbol{g}^{x} \in \mathbb{R}^{n_{x}}, \boldsymbol{g}^{y} \in \mathbb{R}^{n_{y}}$ and $\boldsymbol{g}^{z} \in \mathbb{R}^{n_{z}}$ that consist of the ordered (ascending in our implementation) coordinates (in our setup longitude for $\boldsymbol{g}^{x}$, latitude for $\boldsymbol{g}^{y}$ and altitude for $\boldsymbol{g}^{z}$ ). The elements of the state space vector $\boldsymbol{x}$ are ordered also according to the coordinates, varying fastest in altitude $(z)$ and slowest in latitude $(x)$. Last, three maps $h_{x}, h_{y}$ and $h_{z}$ are needed that map the coordinates of the $i$-th element of $\boldsymbol{x}$ onto the corresponding index of the coordinate in the coordinate vectors $\boldsymbol{g}^{x}, \boldsymbol{g}^{y}$ or $\boldsymbol{g}^{z}$. For example, if the $x$-coordinate of the $i$-th element of $\boldsymbol{x}$ would be the smallest one contained in the grid, the function $h_{x}$ would map $i$ onto the index 0 , as the first element of $\boldsymbol{g}^{x}$ is the smallest X-coordinate. Here, we also use the convention that $g_{i}^{x}$ denotes the $i$-th element of $g^{x}$.

$$
\begin{aligned}
& \text { With } \mathbf{L}_{1}^{x}=\left(l_{i, j}^{x}\right)_{i, j} \text {, then } \\
& l_{i, j}^{x}=\left\{\begin{array}{c}
-1 /\left|\boldsymbol{g}_{h_{x}(i)+1}^{x}-\boldsymbol{g}_{h_{x}(i)}^{x}\right| \\
\text { if } i=j \text { and } h_{x}(i)<n_{x}-1 \\
1 /\left|\boldsymbol{g}_{h_{x}(i)+1}^{x}-\boldsymbol{g}_{h_{x}(i)}^{x}\right| \\
\text { if } h_{x}(i)+1=h_{x}(j), h_{x}(i)<n_{x} \\
-1, h_{y}(i)=h_{y}(j), \text { and } h_{z}(i)=h_{z}(j) \\
0 \text { else }
\end{array}\right.
\end{aligned}
$$

Please note that the absolute value function $|\cdot|$ in this case shall also imply a conversion to kilometers (where applicable), which means that, e.g. the same difference in longitude results in different values for points at different latitudes. The first term describes the main diagonal, the second term a side diagonal. In case of multi-target retrievals, an additional condition must ensure that the entry is zero if $i$ and $j$ describe different target types and a row-scaling must be introduced that introduces different weights for each target type as described by Steck et al. (2005) for 2-D regularization. We found the inverse of the typical standard deviation of a target to be a reasonable value for such a scaling factor. It is also possible to introduce an altitude- or altogether 
location-dependent regularization by scaling the rows of the $\mathbf{L}$ matrices. The matrices $\mathbf{L}_{1}^{y}$ and $\mathbf{L}_{1}^{z}$ can be likewise constructed.

Multiplying the matrix $\mathbf{L}_{1}^{x}$ with $\boldsymbol{x}$ corresponds to computing the partial derivative $\partial / \partial x$ of the atmospheric profile using finite differences using the closest neighboring point. It is straightforward to extend this approach to include higher moments.

\section{Appendix B}

\section{Parametrization of the conjugate gradient scheme}

Combining a Newton scheme with a conjugate gradient method as we describe in Sect. 3.3 exhibits under certain conditions itself regularizing properties (Hanke, 1997). While this in itself may not be desired, it shows some useful properties to reduce the amount of time required for the inversion. While the most computation time in non-linear retrievals is currently used for calculating the Jacobian matrices $\mathbf{F}^{\prime}\left(\boldsymbol{x}_{i}\right)$, test simulations can be executed very efficiently with a linearized retrieval using a fixed, precalculated Jacobian matrix. Further, the conjugate gradient method does not lend itself as easily to efficient parallelization on shared memory machines, implying that spending long time in the iterative solver decreases the overall speed-up factor of the parallelization.

According to Hanke (1995), the regularization properties of the conjugate gradient scheme depend on the number of performed iterations. Further, using only a single iteration corresponds to a steepest descent step while iterating until the error becomes zero corresponds to a Newton step. This shows the similarity to the Levenberg-Marquardt iteration scheme that varies the strength $\lambda$ of a Tikhonov regularization to, in effect, also vary between a steepest descent step and a Newton step (Rodgers, 2000).

To reduce computation time, it is more efficient not to use many iterations in the conjugate gradient scheme to exactly solve the problem posed by each step of the LevenbergMarquardt iteration, but instead to reduce the number of iterations in the conjugate gradient scheme to implicitly introduce a regularization that can replace the LevenbergMarquardt regularization.

"Exact solution" in this sense means that the norm of the error of the solution $\boldsymbol{x}$ to the equation system $\|\mathbf{A} \boldsymbol{x}-\boldsymbol{b}\|_{2}$ is numerically zero, which we chose to mean that the norm is less than $10^{-12}$. We implemented an heuristic that varies the required relative reduction of error $\|\mathbf{A} \boldsymbol{x}-\boldsymbol{b}\|_{2} /\|\boldsymbol{b}\|_{2}$ in the conjugate gradient scheme similarly to how the LevenbergMarquardt parameter is usually varied. That is, it is reduced, if a reduction of the cost function could be achieved or increased in case no reduction could be achieved. The aim is to both reduce the amount of (outer) Newton iterations and (inner) conjugate gradient iterations to save computation time. Similar to Levenberg-Marquardt, it seems obvious that this

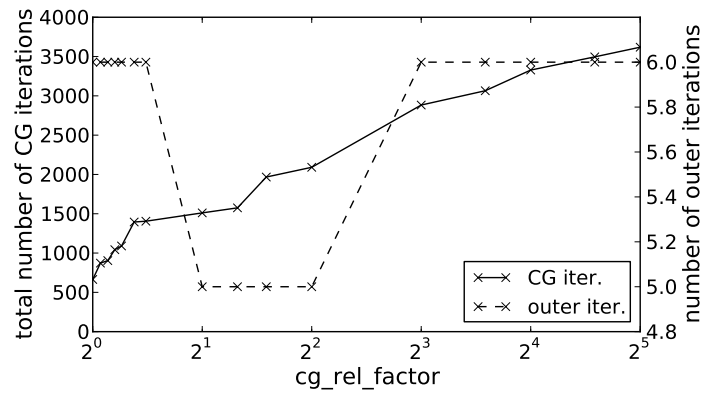

Fig. B1. The effect on parameterizing the conjugate gradient scheme on solving the baseline setup of Sect. 4.2. "cg_rel_factor" describes a multiplicative adjustment factor for modifying the required reduction of the residuum after each outer iteration (increasing the required reduction of the residuum in case the cost function was reduced or vice versa).

scheme does not affect the outcome of the outer iteration if the last iteration requires an exact solution to the equation system. This scheme converges according to Nocedal and Wright (2006, Theorem 7.1) and uses for our problems much less costly evaluations of the forward model than other usual choices based on the norm of the current gradient of the cost function (e.g. Nocedal and Wright, 2006).

Some exemplary results are depicted in Fig. B1. The initial required reduction of the residuum is 0.1 , meaning that the residuum should be reduced until its norm is smaller than $10 \%$ of the norm of the right-hand side. Figure B1 shows how the number of outer and inner iterations changes if the adjustment factor is modified. For an adjustment factor smaller than 1.2, the outer iteration stops with a negligibly larger value of the cost function (with a relative error of $0.01 \%$ ), which could be fixed by adjusting the convergence criterion of the outer iteration. For smaller reduction and adjustment factors than depicted, the number of necessary outer iterations increases significantly. The figure is typical for other initial required reduction values. There is usually an optimum, where the number of outer iterations is minimal while the number of required inner iterations steadily increases.

Acknowledgements. We sincerely thank A. Dudhia, Uni. Oxf., for providing us with the reference forward model (RFM). We also thank the many unmentioned members of the GLORIA team involved in making this instrument a reality.

Edited by: A. Lambert

\section{References}

Carlotti, M., Dinelli, B. M., Raspollini, P., and Ridolfi, M.: Geofit approach to the analysis of limb-scanning satellite measurements, Appl. Optics, 40, 1872-1885, doi:10.1364/AO.40. 001872, 2001.

Carlotti, M., Brizzi, G., Papandrea, E., Prevedelli, M., Ridolfi, M., Dinelli, B. M., and Magnani, L.: GMTR: Two-dimensional 
geo-fit multitarget retrieval model for Michelson Interferometer for Passive Atmospheric Sounding/Environmental Satellite observations, Appl. Optics, 45, 716-727, doi:10.1364/AO.45. $000716,2006$.

Drummond, J. R., Houghton, J. T., Peskett, G. D., Rodgers, C. D., Wale, M. J., Whitney, J., and Williamson, E. J.: The stratospheric and mesospheric sounder on Nimbus 7, Philos. T. Royal Soc. Lond., 296, 219-241, doi:10.1098/rsta.1980.0166, 1980.

Dudhia, A., Morris, P. E., and Wells, R. J.: Fast monochromatic radiative transfer calculations for limb sounding, J. Quant. Spectrosc. Ra., 74, 745-756, doi:10.1016/S0022-4073(01)00285-0, 2002.

Eckermann, S. D., Hoffmann, L., Höpfner, M., Wu, D. L., and Alexander, M. J.: Antarctic NAT PSC belt of June 2003: Observational validation of the mountain wave seeding hypothesis, Geophys. Res. Lett., 36, L02807, doi:10.1029/2008GL036629, 2009.

ESA: Candidate Earth Explorer Core Missions - Report for Assessment: PREMIER- PRocess Exploitation through Measurements of Infrared and millimetre-wave Emitted Radiation, SP-1313/5, ESA Publications Division, ESTEC, Keplerlaan 1, 2200 AG Noordwijk, The Netherlands, 2008.

Fischer, H., Birk, M., Blom, C., Carli, B., Carlotti, M., von Clarmann, T., Delbouille, L., Dudhia, A., Ehhalt, D., Endemann, M., Flaud, J. M., Gessner, R., Kleinert, A., Koopman, R., Langen, J., López-Puertas, M., Mosner, P., Nett, H., Oelhaf, H., Perron, G., Remedios, J., Ridolfi, M., Stiller, G., and Zander, R.: MIPAS: an instrument for atmospheric and climate research, Atmos. Chem. Phys., 8, 2151-2188, doi:10.5194/acp-8-2151-2008, 2008.

Forster, P. M. d. F. and Shine, K. P.: Radiative forcing and temperature trends from stratospheric ozone changes, J. Geophys. Res., 102, 10841-10855, doi:10.1029/96JD03510, 1997.

Francis, G. L., Edwards, D. P., Lambert, A., Halvorson, C. M., Lee-Taylor, J. M., and Gille, J. C.: Forward modeling and radiative transfer for the NASA EOS-Aura High Resolution Dynamics Limb Sounder (HIRDLS) instrument, J. Geophys. Res., 111, D13301, doi:10.1029/2005JD006270, 2006.

Friedl-Vallon, F., Riese, M., Maucher, G., Lengel, A., Hase, F., Preusse, P., and Spang, R.: Instrument concept and preliminary performance analysis of GLORIA, Adv. Space Res., 37, 22872291, doi:10.1016/j.asr.2005.07.075, 2006.

Gille, J. C. and Russel III, J. M.: The Limb Infrared Monitor of the Stratosphere: Experiment Description, Performance, and Results, J. Geophys. Res., 89, 5125-5140, doi:10.1029/ JD089iD04p05125, 1984.

Gordley, L. L. and Russell, J. M.: Rapid inversion of limb radiance data using an emissivity growth approximation, Appl. Optics, 20, 807-813, doi:10.1364/AO.20.000807, 1981.

Gurevich, G.: Application of satellite tomography methods for the improvement of atmospheric sounding inversion algorithms, in: Geoscience and Remote Sensing Symposium, 1995, IGARSS'95, Quantitative Remote Sensing for Science and Applications, International, vol. 2, 1195-1197, doi:10.1109/ IGARSS.1995.521182, 1995.

Hanke, M.: Conjugate gradient type methods for ill-posed problems, John Wiley \& Sons, 1995.

Hanke, M.: Regularizing properties of a truncated Newton-CG algorithm for nonlinear inverse problems, Numer. Func. Anal. Opt., 18, 971-993, doi:10.1080/01630569708816804, 1997.
Hansen, P. C. and O'Leary, D. P.: The Use of the L-Curve in the Regularization of Discrete Ill-Posed Problems, SIAM J. Sci. Comput., 14, 1487-1503, doi:10.1137/0914086, 1993.

Hegglin, M. I., Boone, C. D., Manney, G. L., and Walker, K. A.: A global view of the extratropical tropopause transition layer from Atmospheric Chemistry Experiment Fourier Transform Spectrometer $\mathrm{O}_{3}, \mathrm{H}_{2} \mathrm{O}$, and CO, J. Geophys. Res., 114, D00B11, doi: 10.1029/2008JD009984, 2009.

Höpfner, M., Blom, C., Clarmann, T., Fischer, F., Glatthor, N., Gulde, T., Hase, F., Keim, C., Kimmig, W., Lessenich, K., Piesch, C., Sartorius, C., and Stiller, G.: MIPAS-STR data analysis of APE-GAIA measurements, in: IRS 2000: Current Problems in Atmospheric Radiation; Proc. of the Internat. Radiation Symp., edited by: Smith, W., DEEPAK Publ., 1136-1139, 2001.

Hoffmann, L. and Alexander, M. J.: Retrieval of stratospheric temperatures from Atmospheric Infrared Sounder radiance measurements for gravity wave studies, J. Geophys. Res., 114, D07105, doi:10.1029/2008JD011241, 2009.

Hoffmann, L., Kaufmann, M., Spang, R., Müller, R., Remedios, J. J., Moore, D. P., Volk, C. M., von Clarmann, T., and Riese, M.: Envisat MIPAS measurements of CFC-11: retrieval, validation, and climatology, Atmos. Chem. Phys., 8, 3671-3688, doi:10.5194/acp-8-3671-2008, 2008.

Hoffmann, L., Weigel, K., Spang, R., Schroeder, S., Arndt, K., Lehmann, C., Kaufmann, M., Ern, M., Preusse, P., Stroh, F., and Riese, M.: CRISTA-NF measurements of water vapor during the SCOUT-O3 Tropical Aircraft Campaign, Adv. Space Res., 43(1), 74-81, doi:10.1016/j.asr.2008.03.018, 2009.

Hoor, P., Gurk, C., Brunner, D., Hegglin, M. I., Wernli, H., and Fischer, H.: Seasonality and extent of extratropical TST derived from in-situ CO measurements during SPURT, Atmos. Chem. Phys., 4, 1427-1442, doi:10.5194/acp-4-1427-2004, 2004.

Kaminski, J. W., Neary, L., Struzewska, J., McConnell, J. C., Lupu, A., Jarosz, J., Toyota, K., Gong, S. L., Côté, J., Liu, X., Chance, K., and Richter, A.: GEM-AQ, an on-line global multiscale chemical weather modelling system: model description and evaluation of gas phase chemistry processes, Atmos. Chem. Phys., 8, 3255-3281, doi:10.5194/acp-8-3255-2008, 2008.

Keim, C., Liu, G. Y., Blom, C. E., Fischer, H., Gulde, T., Höpfner, M., Piesch, C., Ravegnani, F., Roiger, A., Schlager, H., and Sitnikov, N.: Vertical profile of peroxyacetyl nitrate (PAN) from MIPAS-STR measurements over Brazil in February 2005 and its contribution to tropical UT NO y partitioning, Atmos. Chem. Phys., 8, 4891-4902, doi:10.5194/acp-8-4891-2008, 2008.

Kiefer, M., Arnone, E., Dudhia, A., Carlotti, M., Castelli, E., von Clarmann, T., Dinelli, B. M., Kleinert, A., Linden, A., Milz, M., Papandrea, E., and Stiller, G.: Impact of temperature field inhomogeneities on the retrieval of atmospheric species from MIPAS IR limb emission spectra, Atmos. Meas. Tech., 3, 14871507, doi:10.5194/amt-3-1487-2010, 2010.

Konopka, P., Grooß, J.-U., Plöger, F., and Müller, R.: Annual cycle of horizontal in-mixing into the lower tropical stratosphere, J. Geophys. Res., 114, 0148-0227, doi:10.1029/2009JD011955, 2009.

Livesey, N., Van Snyder, W., Read, W., and Wagner, P.: Retrieval algorithms for the EOS Microwave limb sounder (MLS), IEEE T. Geosci. Remote, 44, 1144-1155, doi:10.1109/TGRS.2006. 872327, 2006. 
Livesey, N. J. and Read, W. G.: Direct Retrieval of Line-ofSight Atmospheric Structure from Limb Sounding Observations, Geophys. Res. Lett., 27, 891-894, doi:10.1029/1999GL010964, 2000.

Marshall, B. T., Gordley, L. L., and Chu, D. A.: BANDPAK: Algorithms for Modeling Broadband Transmission and Radiance, J. Quant. Spectrosc. Ra., 52, 581-599, doi:10.1016/0022-4073(94) 90026-4, 1994.

Natterer, F.: The mathematics of computerized tomography, Society for Industrial and Applied Mathematics, Philadelphia, PA, USA, 2001.

Nocedal, J. and Wright, S. J.: Numerical Optimization, Springer, 2006

Nolet, G.: Solving or resolving inadequate and noisy tomographic systems, J. Comput. Phys., 61, 463-482, doi:10.1016/ 0021-9991(85)90075-0, 1985.

Nolet, G.: A Breviary Of Seismic Tomography: Imaging The Interior Of The Earth And Sun, Cambridge University Press, 2008.

Offermann, D., Grossmann, K.-U., Barthol, P., Knieling, P., Riese, M., and Trant, R.: Cryogenic Infrared Spectrometers and Telescopes for the Atmosphere (CRISTA) experiment and middle atmosphere variability, J. Geophys. Res., 104, 16311-16325, doi: 10.1029/1998JD100047, 1999.

Remedios, J. J., Leigh, R. J., Waterfall, A. M., Moore, D. P., Sembhi, H., Parkes, I., Greenhough, J., Chipperfield, M. P., and Hauglustaine, D.: MIPAS reference atmospheres and comparisons to V4.61/V4.62 MIPAS level 2 geophysical data sets, Atmos. Chem. Phys. Discuss., 7, 9973-10017, doi:10.5194/acpd7-9973-2007, 2007.

Riese, M., Preusse, P., Spang, R., Ern, M., Jarisch, M., Grossmann, U., and Offermann, D.: Measurements of trace gases by the cryogenic infrared spectrometers and telescopes for the atmosphere CRISTA experiment, Adv. Space Res., 19, 563-566, doi:10.1016/S0273-1177(97)00172-5, 1997.

Riese, M., Manney, G. L., Oberheide, J., Tie, X., Spang, R., and Küll, V.: Stratospheric transport by planetary wave mixing as observed during CRISTA-2, J. Geophys. Res., 107, 8179, doi: 10.1029/2001JD000629, 2002.

Riese, M., Friedl-Vallon, F., Spang, R., Preusse, P., Schiller, C., Hoffmann, L., Konopka, P., Oelhaf, H., von Clarmann, T., and Höpfner, M.: GLObal limb Radiance Imager for the Atmosphere (GLORIA): Scientific objectives, Adv. Space Res., 36, 989-995, doi:10.1016/j.asr.2005.04.115, 2005.

Rodgers, C. D.: Inverse Methods for Atmospheric Sounding: Theory and Practice, vol. 2 of Series on Atmospheric, Oceanic and Planetary Physics, World Scientific, 2000.

Roewe, D. A., Gille, J. C., and Bailey, P. L.: Infrared limb scanning in the presence of horizontal temperature gradients: an operational approach, Appl. Optics, 21, 3775-3783, doi:10.1364/AO. 21.003775, 1982

Saad, Y.: Iterative Methods for Sparse Linear Systems, S.I.A.M., Philadelphia, 2nd edition, 2003.

Schiller, C., Grooß, J.-U., Konopka, P., Plöger, F., Silva dos Santos, F. H., and Spelten, N.: Hydration and dehydration at the tropical tropopause, Atmos. Chem. Phys., 9, 9647-9660, doi:10.5194/acp-9-9647-2009, 2009.
Solomon, S., Rosenlof, K. H., Portmann, R. W., Daniel, J. S., Davis, S. M., Sanford, T. J., and Plattner, G.-K.: Contributions of Stratospheric Water Vapor to Decadal Changes in the Rate of Global Warming, Science, 327, 1219-1223, doi:10.1126/ science.1182488, 2010.

Solomon, S. C., Hays, P. B., and Abreu, V. J.: Tomographic inversion of satellite photometry, Appl. Optics, 23, 3409-3414, doi: 10.1364/AO.23.003409, 1984.

Spang, R., Hoffmann, L., Kullmann, A., Olschewski, F., Preusse, P., Knieling, P., Schroeder, S., Stroh, F., Weigel, K., and Riese, M.: High resolution limb observations of clouds by the CRISTA-NF experiment during the SCOUT-O3 tropical aircraft campaign, Adv. Space Res., 42, 1765-1775, doi:10.1016/j.asr.2007.09.036, 2008.

Steck, T. and von Clarmann, T.: Constrained profile retrieval applied to the observation mode of the Michelson Interferometer for Passive Atmospheric Sounding, Appl. Optics, 40, 35593571, doi:10.1364/AO.40.003559, 2001.

Steck, T., Höpfner, M., von Clarmann, T., and Grabowski, U.: Tomographic retrieval of atmospheric parameters from infrared limb emission observations, Appl. Optics, 44, 3291-3301, doi: 10.1364/AO.44.003291, 2005.

Stevenson, D. S., Dentener, F. J., Schultz, M. G., Ellingsen, K., van Noije, T. P. C., Wild, O., Zeng, G., Amann, M., Atherton, C. S., Bell, N., Bergmann, D. J., Bey, I., Butler, T., Cofala, J., Collins, W. J., Derwent, R. G., Doherty, R. M., Drevet, J., Eskes, H. J., Fiore, A. M., Gauss, M., Hauglustaine, D. A., Horowitz, L. W., Isaksen, I. S. A., Krol, M. C., Lamarque, J.-F., Lawrence, M. G., Montanaro, V., Pitari, G., Prather, M. J., Pyle, J. A., Rast, S., Rodriguez, J. M., Sanderson, M. G., Savage, N. H., Shindell, D. T., Strahan, S. E., Sudo, K., and Szopa, S.: Multimodel ensemble simulations of present-day and near-future tropospheric ozone, J. Geophys. Res., 111, 0148-0227, doi:10.1029/2005JD006338, 2006.

Struzewska, J. and Kaminski, J. W.: Formation and transport of photooxidants over Europe during the July 2006 heat wave observations and GEM-AQ model simulations, Atmos. Chem. Phys., 8, 721-736, doi:10.5194/acp-8-721-2008, 2008.

Tarantola, A.: Inverse Problem Theory, Society for Industrial and Applied Mathematics, Philadelphia, 2004.

Ungermann, J., Hoffmann, L., Preusse, P., Kaufmann, M., and Riese, M.: Tomographic retrieval approach for mesoscale gravity wave observations by the PREMIER Infrared Limb-Sounder, Atmos. Meas. Tech., 3, 339-354, doi:10.5194/amt-3-339-2010, 2010.

von Clarmann, T., De Clercq, C., Ridolfi, M., Höpfner, M., and Lambert, J.-C.: The horizontal resolution of MIPAS, Atmos. Meas. Tech., 2, 47-54, doi:10.5194/amt-2-47-2009, 2009.

Weigel, K., Riese, M., Hoffmann, L., Hoefer, S., Kalicinsky, C., Knieling, P., Olschewski, F., Preusse, P., Spang, R., Stroh, F., and Volk, C. M.: CRISTA-NF measurements during the AMMASCOUT-O3 aircraft campaign, Atmos. Meas. Tech., 3, 14371455, doi:10.5194/amt-3-1437-2010, 2010.

Weinreb, M. P. and Neuendorffer, A. C.: Method to Apply Homogeneous-path Transmittance Models to Inhomogenous Atmospheres, J. Atmos. Sci., 30, 662-666, doi:10.1175/ 1520-0469(1973)030\$〈\$0662:MTAHPT\$ \$2.0.CO;2, 1973. 